Draft VERSion SEPTEMBER 13, 2021

Preprint typeset using $\mathrm{LAT}_{\mathrm{E}} \mathrm{X}$ style emulateapj v. 2/19/04

\title{
THE COMPLEX NORTH TRANSITION REGION OF CENTAURUS A: A GALACTIC WIND
}

\author{
Susan G. NeFF \\ NASA's Goddard Space Flight Center, Laboratory for Observational Cosmology, Mail Code 665, Greenbelt, Maryland, 20771
}

JEAN A. EILEK

Physics Department, New Mexico Tech, Socorro NM 87801 and

National Radio Astronomy Observatory*†, Socorro NM 87801

Frazer N. OWEN

National Radio Astronomy Observatory*, Socorro NM 87801

Draft version September 13, 2021

\begin{abstract}
We present deep GALEX images of NGC 5128, the parent galaxy of Centaurus A. We detect a striking "weather ribbon" of Far-UV and $\mathrm{H} \alpha$ emission which extends more than $35 \mathrm{kpc}$ northeast of the galaxy. This ribbon is associated with a knotty ridge of radio/X-ray emission and is an extension of the previously known string of optical emission-line filaments. Many phenomena in the region are too short-lived to have survived transit out from the inner galaxy; something must be driving them locally. We also detect Far-UV emission from the galaxy's central dust lane. Combining this with previous radio and Far-IR measurements, we infer an active starburst in the central galaxy which is currently forming stars at $\sim 2 M_{\odot} \mathrm{yr}^{-1}$, and has been doing so for 50-100 Myr. If the wind from this starburst is enhanced by energy and mass driven out from the AGN, the powerful augmented wind can be the driver needed for the northern weather system. We argue that both the diverse weather system, and the enhanced radio emission in the same region, result from the wind's encounter with cool gas left by one of the recent merger/encounter events in the history of NGC 5128.
\end{abstract}

Subject headings: galaxies: active - galaxies: individual (NGC 5128, Centaurus A) - galaxies: jets galaxies: starburst - galaxies: winds - radio continuum: galaxies

\section{INTRODUCTION}

The radio source Centaurus A ("Cen A") and its parent galaxy, NGC 5128 are the nearest active-galaxy system. At a distance of only $3.8 \mathrm{Mpc}$, (Harris et al. 2010; $1^{\prime} \simeq 1.14 \mathrm{kpc}$ ), both the radio source Cen A and the galaxy NGC5128 can be scrutinized with a sensitivity and resolution impossible for other active galaxies.

NGC 5128 is fundamentally a normal elliptical galaxy, dominated by an old stellar population, with kinematic signatures typical of other massive ellipticals (e.g., Peng et al. 2004; Woodley et al. 2010; Rejkuba et al. 2011). However, the galaxy's optical appearance is dominated by the unusual, and iconic, dust band (Dunlop 1828, Herschel 1847). The central dust band is the site of vigorous and ongoing star formation. In this paper, we show that the extended starburst is strong enough to drive out a wind.

On larger scales, a striking complex of optical emissionline gas, active star formation, cold gas and dust clouds, and overpressured radio and X-ray knots extends into the outer reaches of the galaxy, $\sim 10-35 \mathrm{kpc}$ to the NE (all distances are given in projection on the sky). This system is spatially coincident with the diffuse radio emission we described in Neff, Eilek, and Owen (2015; Paper 1). We

Electronic address: susan.g.neff@nasa.gov

* The National Radio Ástronomy Ubservatory is a facility of the National Science Foundation operated under cooperative agreement by Associated Universities Inc.

$\dagger$ Adjunct Astronomer at the National Radio Astronomy Observatory. suggest in this paper that both structures are causally related to an energy-carrying flow in the region.

As in Paper 1, our focus here is the inner $\sim 50 \mathrm{kpc}$ of the Cen A / NGC 5128 system. We present new GALEX observations and consider what they, together with new radio data (Paper 1), reveal about the astrophysics of this region. In the rest of this section we present an introduction to key aspects of the system. In Table 1 we list regions and features discussed throughout the paper, and give approximate distances from the galaxy's center.

\subsection{Large and small-scales}

The outer radio lobes - The radio source Cen A was first identified with the galaxy NGC 5128 by the radio emission from its Outer Lobes (Bolton et al. 1949), which extend $\sim 600 \mathrm{kpc}$ end-to-end on the sky (e.g., Junkes et al. 1993; Feain et al. 2011). The outer radio lobes are also detected as extended $\gamma$-ray sources (Abdo et al. 2010), thought to result from inverse Compton scattering of Cosmic Microwave Background photons. Eilek (2014) argues that the dynamical age of Cen A's outer lobes is on the order of $\sim 1 \mathrm{Gyr}$ (assuming the lobes lie at some finite angle to the sky plane), but that they must have been last energized no more than $\sim 30$ Myr ago to keep shining in radio and $\gamma$-rays. Any energy supplied to the Outer Lobes by the inner galaxy must, of course, have moved through the middle ( 50-kpc scale) regions which we study in this paper. 
TABLE 1

Important REGIONS \& FEATURES IN CEN A NGC 5128

\begin{tabular}{|c|c|}
\hline Definition & $\begin{array}{r}\text { Scale }^{\mathrm{a}} \\
(\mathrm{kpc})\end{array}$ \\
\hline (North, South) Inner radio Lobes (N/S IL) & $\sim 7, \sim 5.5$ \\
\hline North Middle (radio) Lobe ${ }^{\mathrm{b}}$ (NML) & $10-40$ \\
\hline (North, South) Transition Regions (N/S TR) & $10-40$ \\
\hline (North, South) Outer radio Lobes (N/S OL) & $40-300$ \\
\hline Weather Ribbon / Weather System & $10-40$ \\
\hline Inner Filament $^{\mathrm{c}}$, Outer Filament ${ }^{\mathrm{c}}$ & 8,15 \\
\hline
\end{tabular}

a Spatial scales give approximate distance from AGN, in projection. b We use "North Middle Lobe" to refer only to the radio-loud part of the North Transition Region.

c The terms "Inner Filament" and "Outer Filament" are well established in the literature, and refer to specific, optically-bright emission line filaments.

The central AGN - The massive black hole in the nucleus of NGC 5128 is currently active, as indicated by pc-scale radio jets, a northern few-kpc-scale radio and $\mathrm{X}$-ray jet, and inner lobes extending $\sim 7 \mathrm{kpc} \mathrm{NE}$ and $\sim 5.5 \mathrm{kpc}$ SW of the galactic core (e.g., Burns et al.1983). Both brightness and proper motion asymmetries of the inner jet (e.g., Tingay et al.2001) and Faraday depolarization of the South Inner Lobe (Clarke et al. 1992) suggest that the northern jet is approaching us while the South Inner Lobe is aimed away from us. X-ray observations of a shock driven into the ISM by the Southern Inner Lobe show the Inner Lobes are only $\sim 1-2$ Myr old (Croston et al. 2009, Paper 1). This suggests the Active Galactic Nucleus (AGN) has only recently restarted as a collimated outflow. The power currently produced by the AGN, several $\times 10^{43} \mathrm{erg} \mathrm{s}^{-1}$, is interestingly close to that required to power the outer lobes over their $\sim 1$ Gyr lifetime (Eilek 2014, paper 1).

The central disk and inner galaxy - The iconic dust lane in NGC 5128 is part of a thin $(\sim 250 \mathrm{pc})$, multiply warped gas and dust disk. It is a site of active star formation. UBV photometry of young stars (van den Bergh 1976, Dufour et al. 1979) and young clusters (Minitti et al. 2004), together with $\mathrm{H} \alpha$ and [NII] imaging of HII regions (Bland et al. 1987) and Far-Infrared (FIR) spectroscopy of the disk (Unger et al.2000), indicate ongoing active star formation in the disk that has continued for at least $50 \mathrm{Myr}$. The disk is thought to result from a merger or stripping interaction, between a large elliptical galaxy and a smaller gas-rich system, that occurred 250-750 Myr ago (Struve et al. 2010, Sparke et al. 1996, Quillen et al. 1993).

\subsection{Intermediate scales: the transition regions}

We refer to the regions between $\sim 10-40 \mathrm{kpc}$ north and south of the galaxy as the North and South Transition Regions (NTR, STR). These regions are outside the main body of the galaxy, beyond the restarted Inner radio Lobes, extending approximately into the start of the Outer radio Lobes. While the southern transition region is unremarkable, the northern transition region hosts a collection of unusual features, as follows.

\subsubsection{Radio emission: the "North Middle Lobe"}

The North Transition Region is much brighter in the radio than the equivalent region to the south. The radiobright northern region, which we studied in Paper 1, is called the "North Middle Lobe" (NML) in the literature. In addition to the extended, diffuse radio emission, Morganti et al. (1999) identified a linear radio structure in the NML, and suggested that it might be a "large-scale jet" connecting the inner and outer radio lobes. However, in Paper 1 we showed that the linear feature does not appear to be a radio jet. Rather, it is part of a knotty, radio-bright ridge embedded in the larger, overpressured, diffuse structure of the NML.

The North Middle Lobe seems to have pushed aside the hot Interstellar Medium (ISM) of NGC 5128; X-ray images reveal a cavity north of the galaxy, which is approximately coincident with the NML (e.g., Kraft et al.2009; Paper 1). We showed in Paper 1 that the radio-loud North Middle Lobe also needs ongoing energization: without a driver, the NML will fade in $\lesssim 20$ Myr due to expansion and high-frequency synchrotron losses.

\subsection{2. "Weather" in the north transition region}

In addition to unusual radio emission and the disturbed galactic ISM, the North Transition Region hosts an X-rayloud filament with embedded clouds, optical emission-line filaments, recently formed stars, and a broken ring of cold atomic and molecular gas - all in close proximity. As noted in Paper 1, we refer to this complex mixture of radio emission, disturbed multiphase ISM, and star formation as the "weather system".

Emission-line filaments and star formation- A trail of emission line filaments, young star clusters, and Ultraviolet emission (UV, $\lambda 1344-3000 \AA$ ) stretches outward from the galaxy. Most previous optical work has focused on two bright regions close the galaxy, known as the "Inner" and "Outer" Filaments. The Inner Filament is $\sim 8 \mathrm{kpc}$ from the core, while the Outer Filament is $\sim 15 \mathrm{kpc}$ from the core. Both Filaments contain ionized gas, with high internal/turbulent velocities ( $\gtrsim 100 \mathrm{~km} \mathrm{~s}^{-1}$, (e.g., Graham 1998), and so should notlast more than 10-15 Myr. Both regions also contain young stars, with ages $\lesssim 10$ Myr (e.g., Mould et al. 2000, Crockett et al. 2011), thus recent and ongoing star formation. In this paper, we show that an extended "weather ribbon" of UV and $\mathrm{H} \alpha$ emission extends much further, reaching from the North Inner Lobe to at least $\sim 35 \mathrm{kpc}$ from the galaxy's core (see also Graham \& Price 1981).

Knotty radio/X-ray ridge - The knotty, radio-bright ridge reported in Paper 1 sits close to the SE edge of the North 
Middle Lobe. It also sits very close to a similar, knotty, Xray bright ridge (Feigelson et al.1981, Kraft et al. 2009). We showed in paper 1 that the radio knots and X-ray knots are spatially close to each other, but not exactly coincident; both sets of knots are $\sim 1 \mathrm{kpc}$ in size and - if they are diffuse plasma - are at $\gtrsim 20$ times higher pressure than their surroundings. In this paper we show that the knotty radio/X-ray ridge is also nearly coincident with the outer part of the string of young stars and emission-line filaments mentioned above.

Cold gas and dust - The Transition Regions also contain clouds of dusty, cold gas (e.g., Schiminovich et al. 1994), which form a broken ring orbiting NGC 5128. CO emission and warm dust have been detected from the densest parts of the HI clouds (Charmandaris et al. 2000, Auld et al. 2012). One edge of the outermost HI cloud appears spatially coincident with the Outer Filament and with the radio and X-ray ridges. Osterloo \& Morganti (2005) found that the cloud edge shows anomalous, turbulent velocities, and speculate that gas stripped from the cloud provides seed material for the emission line filaments NE of the cloud.

\subsection{Models for the transition regions}

The astrophysics of the transition regions, as a whole, is not well understood, although various models have been proposed for specific phenomena there.

Star formation and emission line filaments - There is nearconsensus in the literature that an outflow must be causally related to the emission-line clouds and young stars in the weather ribbon NE of the galaxy. Many authors assume the narrow feature seen by Morganti et al. (1999) is a collimated jet which has energized the lineemitting clouds and induced star formation in the ribbon. Detailed models include weak shocks in dense clouds photoionizing the ambient gas (Sutherland et al.1993) and shocks causing cloud fragmentation and collapse leading to star formation (e.g., Fragile et al.2004, Croft et al. 2006). However, we showed in Paper 1 that the linear feature does not have the properties of a radio jet. Although the lack of a detected jet seems to challenge the jet-driven shock models, we argue in this paper that a broad wind outflow can play the same role as a narrow jet in energizing the clouds and inducing star formation.

Radio loudness of the North Middle Lobe - We noted in Paper 1 that models proposed to explain the radio-loudness of the North Middle Lobe have either assumed the presence of a radio jet through the region, or attributed the NML to a slowly rising, bouyant bubble. We argued there that none of these models can explain all observed properties of the region - in particular the short-lived nature of several radio-related phenomena, the need for ongoing energy input to both Outer Lobes, and the differences in radio properties between the North and South Transition Regions. We suggest in this paper that a broad wind outflow can explain the radio properties of the North Middle
Lobe, just as it can energize the emission-line clouds and drive star formation in the weather ribbon.

\subsection{Organization of this paper}

In this paper, we present Galaxy Evolution Explorer $(G A L E X)$ observations of the inner $\sim 50 \mathrm{kpc}$ of Cen A/NGC 5128, and consider the likely astrophysics of the Transition Regions. In Section 2 we present new GALEX observations which reveal an extended ribbon of UV emission, extending well into the North Transition Region, as well as an active starburst in the central disk. In Section 3 we describe the complexities of the weather system, and show that nearly all of its features require ongoing energy input. In Section 4 we combine our FUV measurements with results from the literature to estimate the strength of the starburst in the dusty disk. We argue that a wind from this starburst - probably enhanced by energy input from the AGN - is flowing through the North and South Transition regions at present. In Section 5 we explore a simple model of the wind. In Section 6 we argue that the wind can drive the weather system and enhance the radio brightness of the northern transition region. Finally, in Section 7 we summarize our results.

\section{GALEX OBSERVATIONS}

UV observations made by GALEX reveal a continuous UV-emitting ribbon extending more than $30^{\prime}$ to the NE of the galaxy center, and show that the dust disk of NGC 5128 is a strong UV source.

GALEX is a NASA Small Explorer mission optimized for wide-field ultraviolet imaging (Martin et al. 2005, Morrissey et al. 2005). GALEX images cover a $1.25^{\circ}$ diameter circular field, with an angular resolution of $\sim 5^{\prime \prime}$. FarUV (FUV, $\lambda 1344-1786 \AA \AA^{\circ}, \lambda_{e f f}=1539 \AA$ ) and Near-UV (NUV, $\left.\lambda 1771-2831, \stackrel{\circ}{A}, \lambda_{e f f}=2316 \stackrel{\circ}{A}\right)$ images are obtained simultaneously, using a dichroic beamsplitter and two photon-counting detectors. Science observations are only made during the orbital nighttime.

Cen A was observed by GALEX for 14 eclipses between 02-04 April 2004 and 01 May - 02 June 2008 in both FUV and NUV, and for a further 13 eclipses in NUVonly between 02-09 April 2005. Each exposure was processed using the standard GALEX pipeline (Morrissey et al. 2007) to reconstruct the image from photon lists, to un-dither the image, remove optical distortions and non-uniform detector response, to remove internal reflections from nearby bright stars, and to fit and subtract the sky background. Individual background-subtracted images were then added to produce final combined images (total exposure time of $20072 \mathrm{sec}$ in FUV, $30428 \mathrm{sec}$ in NUV), which are available from MAST. ${ }^{1}$

For this study, we extracted subimages including the galaxy and most of the North Middle Lobe detected in our radio observations (Paper 1 ). The subimages were smoothed by a box $7.5^{\prime \prime}$ on a side, to enhance the faint

\footnotetext{
1 The Mikulski Archive for Space Telescopes; GALEX data available at galex.stsci.edu .
} 


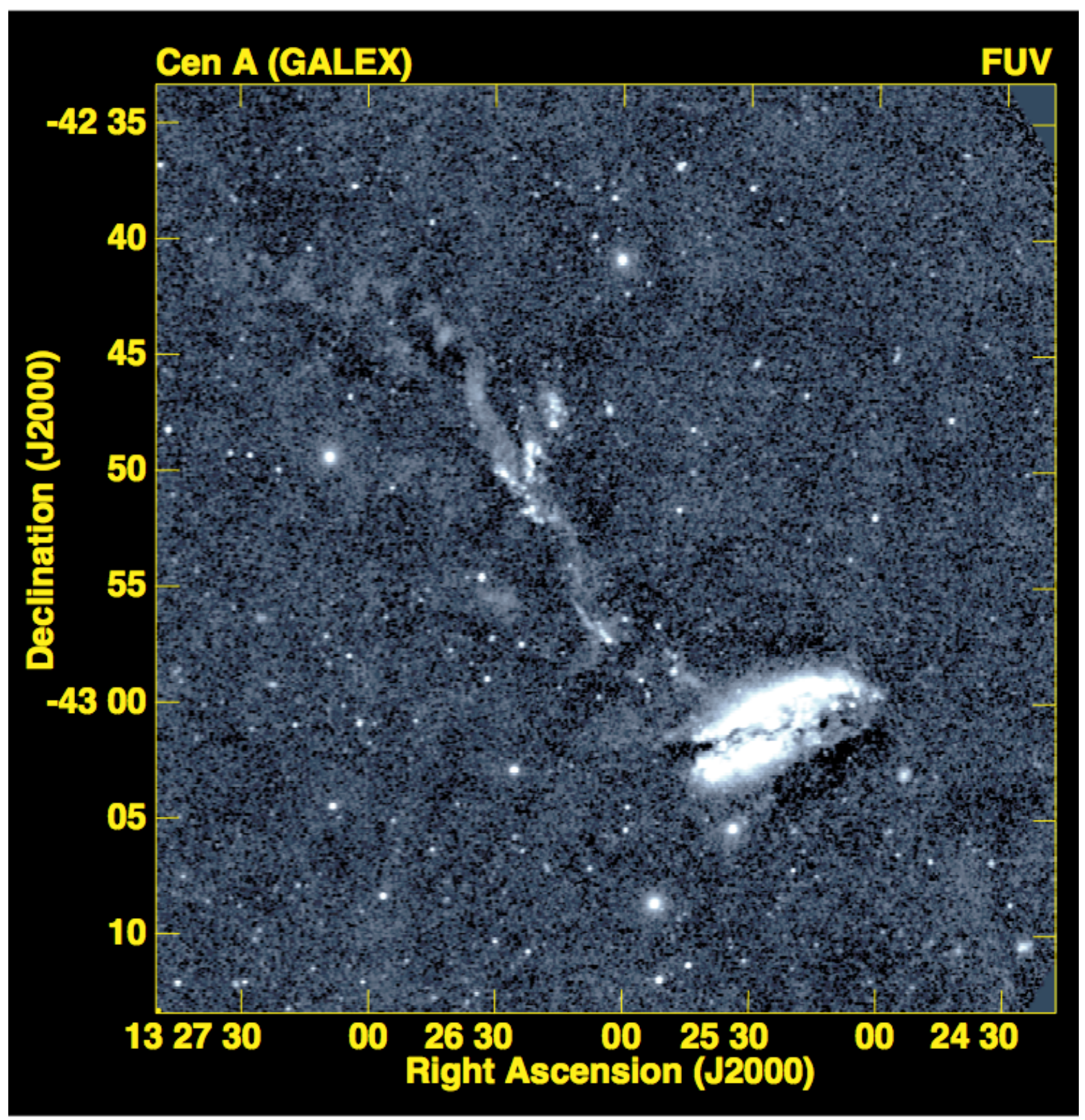

FIG. 1.- FUV image (from GALEX: $\lambda_{e f f}=1539 \AA$ ) of the region including NGC 5128 and the North Transition Region. The tilted central gas/dust disk is clearly seen; FUV emission extends at least $1.5^{\prime}$ above and below the dust lane, and indicates a strong starburst in the disk. The bright "Inner" and V-shaped "Outer" Filaments are also prominent in this image. A fainter, twisted ribbon of FUV emission extends out at least $\sim 35^{\prime}(\sim 40 \mathrm{kpc})$ from the center, and includes the Inner and Outer filaments. This image has been smoothed by a $7.5^{\prime \prime}$ box to highlight diffuse emission.

UV emission, and for comparison with the much lower resolution radio images. In Figure 1 we show the deep GALEX FUV continuum image of a $40^{\prime}$ (44 kpc) field around NGC 5128. The NUV image is similar in appearance but is dominated by foreground stars, making it more difficult to see the faint extended emission.

Figure 1 shows that a dramatic line of FUV emission extends more than $30^{\prime}(\sim 35 \mathrm{kpc})$ NE from the galaxy center, into the weather system in the North Transition Region. This line of FUV emission appears to be a twisted curving ribbon; it includes, but goes well beyond, the previously known Inner and Outer filaments. We do not de- tect any corresponding extended FUV emission SW of the galaxy. (Diffuse NUV emission may extend $\sim 15^{\prime}(\sim 17$ kpc) SW of the galaxy (Auld et al. 2012), but it is right at the edge of the GALEX detector and is challenging to display.)

In Figure 2 we show the relationship of the UV emission to radio emission in the North Transition Region. We see that the twisted FUV ribbon sits close to, but inside of, the SE edge of the diffuse radio emission; it is also approximately coincident with the knotty radio-loud ridge (as indicated by the three radio knots, and as discussed in Section 3.2). The diffuse radio emission of the NML 


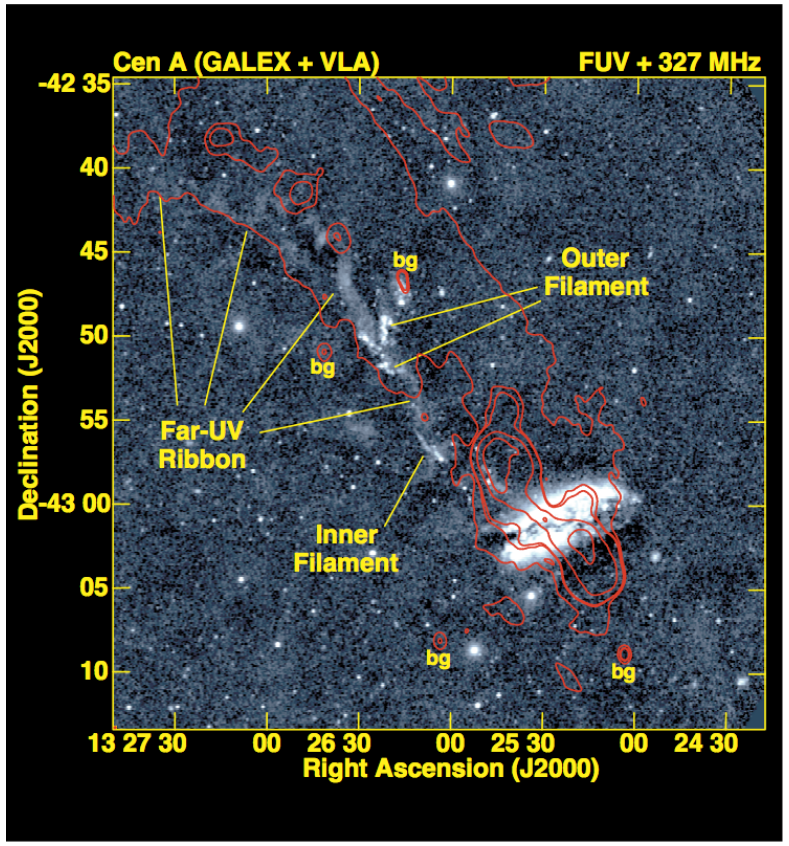

Fig. 2.- Overlay of $327 \mathrm{MHz}$ NML radio emission from Paper 1 (contours), superposed on the FUV GALEX image. Radio contours are at $0.07,0.15,0.18,2.0,10.0 \mathrm{Jy} /$ beam. Key optical/FUV features are labelled, as are four background radio sources ("bg") unrelated to Cen A. The extended FUV ribbon overlays the radio ridge running through the eastern edge of the diffuse radio emission, including the radio knots. Diffuse NML emission continues to the $\mathrm{N}$ and NW of this image, but is not imaged well in these observations. The gap in the diffuse radio emission, approximately coincident with the Inner Filament, is real; the $327-\mathrm{MHz}$ emission from this region is much fainter than from the rest of the NML.

extends $\gtrsim 20^{\prime}(\sim 22 \mathrm{kpc}) \mathrm{NW}$ of the ridge as it connects to the North Outer Lobe; similar behavior is apparent at higher frequencies (1.4 GHz, Morganti et al. 1999 and Feain et al. 2011; $4.8 \mathrm{GHz}$, Junkes et al. 1993). However, we see no sign of corresponding diffuse FUV emission in this region; the FUV we detect is localized in the weather ribbon.

Figure 1 also shows that the central disk of NGC5128 is a strong FUV source. Because the FUV bandpass of GALEX is most sensitive to $\mathrm{O}$ and $\mathrm{B}$ stars, it does an excellent job of identifying regions of recent star-formation. This image reveals the bright (overexposed) star-forming disk associated with the central starburst, along with the well-known dust lane cutting across the tilted disk. We see FUV emission above and below, as well as along, the central dust lane. In Hubble Space Telescope images of the central $\sim 6$ pc of the disk (Marconi et al. 2000), individual young stars and clusters can be identified. However, at the GALEX resolution $\left(\sim 5^{\prime \prime}\right)$, we are not able to tell how much of the FUV emission is direct starlight and how much is scattered.

\section{THE WEATHER SYSTEM: PROBING THE NORTH TRANSITION REGION}

The unusual, extended weather system north of NGC 5128 contains a complex mix of young stars, emissionline clouds, diffuse radio emission and a knotty ridge of
radio/X-ray emission. The collection of interesting oddities in the North Transition Region can be confusing. We show the relationship of the various components making up the weather system in Figure 3 . The various constituents of the weather system can provide important, if indirect, probes of the environment in the North Transition Region. As mentioned in Sections 1.2 and 1.3 , none of the models so far proposed to explain these diverse phenomena works well for all of them.

In this section we discuss the properties and possible origins of the various features in the weather system. We find that many of the interesting exotica are likely to have quite short lifetime, and to require very recent, probably in-situ re-energizing. We touch on how the weather phenomena might be related to a wind driven by the central starburst. In following sections we explore the likely galactic wind in more detail, and show how a wind can drive many, if not all, of the observed weather features.

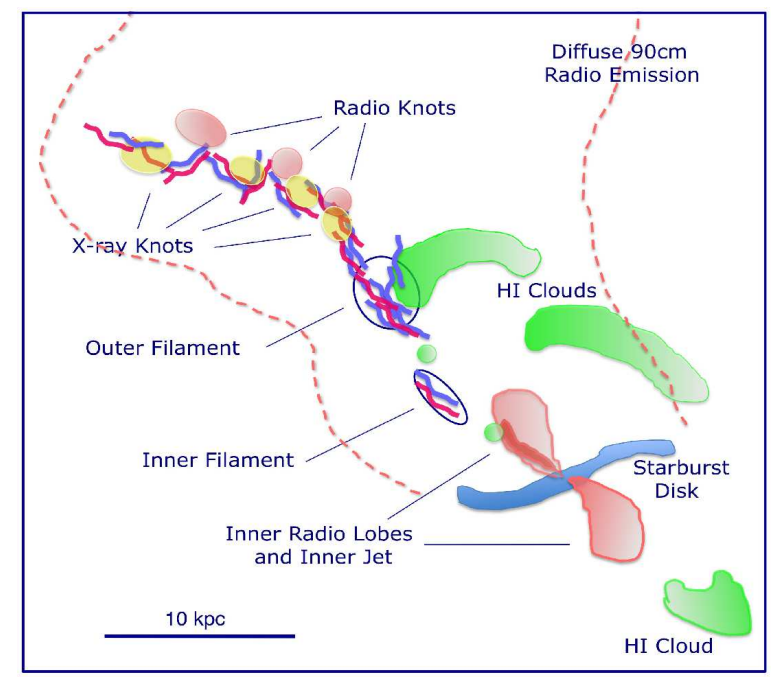

FIG. 3.- Schematic diagram, showing the components of the "weather system" in the North Transition Regon of Cen A, and their apparent relationship to the central galaxy. Each component is discussed in Section 3 Blue and red wiggly lines indicate FUV emission and $\mathrm{H} \alpha$ filaments, respectively.

\subsection{Warm clouds and young stars}

FUV and Ho ribbon - Both optical and FUV data reveal a complex mix of young stars, active star formation and emission-line gas extending throughout the $\sim 30 \mathrm{kpc}$ eastern part of the NTR. Figure 4 shows a side-by-side comparison of $\mathrm{H} \alpha$ and FUV images of the weather system. The left panel shows part of an $\mathrm{H} \alpha$ image, taken by I. Evans and A. Koratkar, using the MOSAIC2 camera on the $4 \mathrm{~m}$ Blanco telescope at CTIO. The right panel of Figure 4 shows the same field, extracted from our full-field FUV image (Figure 1). The FUV and $\mathrm{H} \alpha$ emission appear to be coincident, with the same extent and the same morphology.

The FUV/H $\alpha$ ribbon extends over $25^{\prime}$ (28 kpc) starting near the galaxy center and falling below our detection 

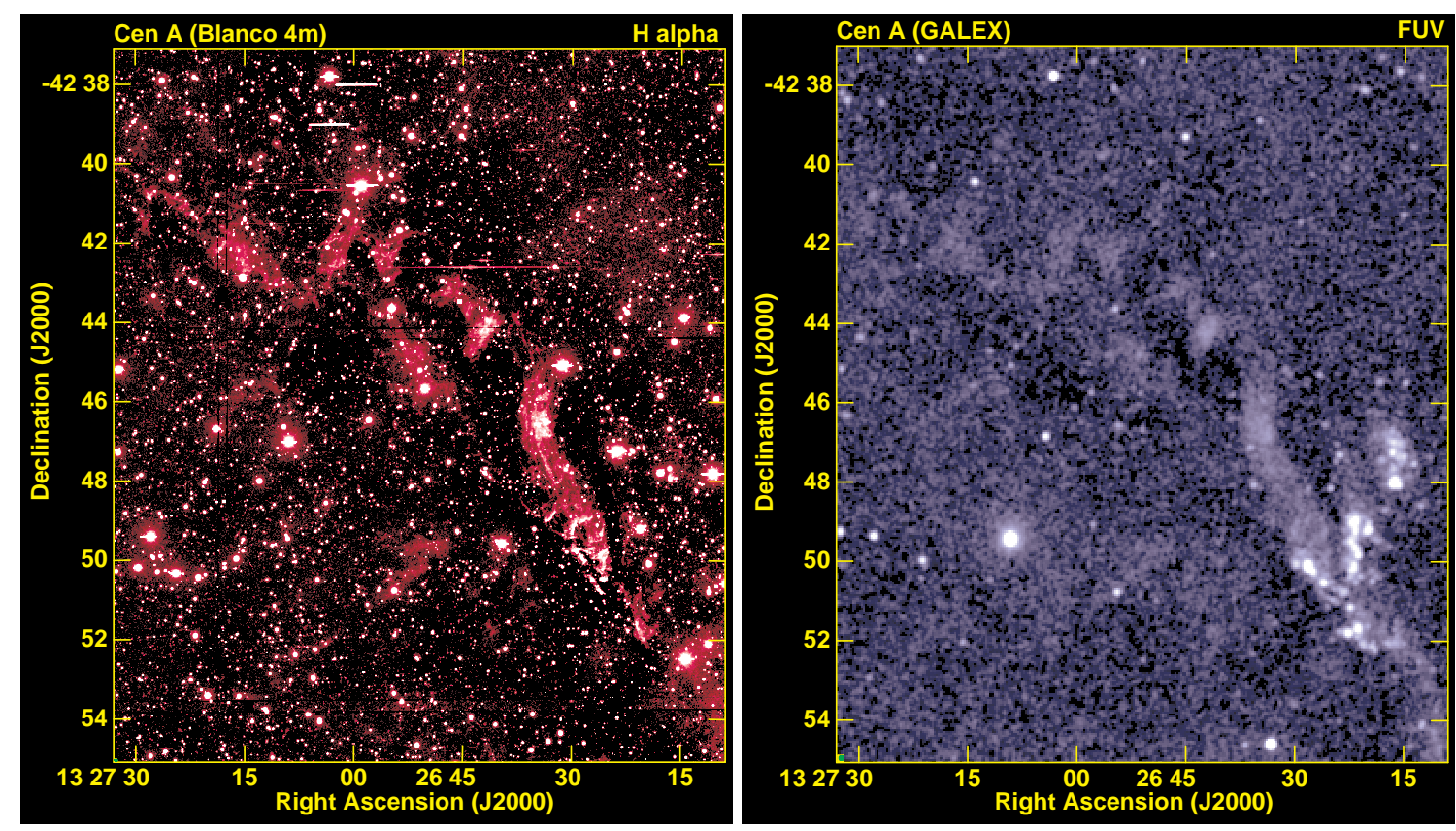

Fig. 4. - The FUV weather ribbon, as seen in $\mathrm{H} \alpha$ and FUV emission. Left: an $\mathrm{H} \alpha$ image of the weather ribbon, kindly provided by I. Evans and A. Koratkar. A $1500 \mathrm{sec}$ exposure, using a narrow-band interference filter centered at $6009 \AA$, in $\sim 1^{\prime \prime}$ seeing. It has been smoothed with a $1.2^{\prime \prime}$ gaussian and then resampled with $1.5^{\prime \prime}$ pixels to match the FUV image. A background gradient due to the host galaxy has been subtracted, using a 199-pixel smoothing window $\left(\sim 300^{\prime \prime}\right)$ to produce the subtracted background. Right, extracting the same field from Figure 1 still with 7.5" smoothing to bring out the faint emission. Comparison shows that the diffuse FUV and H $\alpha$ ribbons are nearly identical, extending from close to the galaxy (below and right of this image), through the V-shaped Outer Filament (more easily seen in the FUV image, right), and continuing in a twisted-ribbon structure to $33^{\prime}(\sim 37 \mathrm{kpc})$ from the galaxy center.

levels $\sim 33^{\prime}$ (37kpc) from the galaxy center. The comparison makes it clear that both $\mathrm{H} \alpha$ and FUV emission extend well beyond the bright emission line filaments previously known (Inner and Outer Filaments, indicated in Figures 2 and 3). The FUV/H $\alpha$ ribbon contains the well-studied Inner and Outer Filaments, as well as $\mathrm{H} \alpha$ emission regions farther away from the galaxy which were examined by Graham \& Price (1981) ${ }^{2}$. FUV and $\mathrm{H} \alpha$ emission, apparently part of the same large structure, also extends inward from the fields shown in Figure 4 towards the galaxy center (starting $\lesssim 7^{\prime}(8 \mathrm{kpc})$ from the galaxy center, as can be seen in Figure 1).

Kinematics - The gas in the Inner and Outer Filaments, and in a few brighter regions in the outer ribbon, has a complex velocity structure (Graham \& Price 1981; Morganti et al.1991; Graham 1998). The velocity of the filaments in the line of sight is $\sim 300-400 \mathrm{~km} \mathrm{~s}^{-1}$ away from the galaxy and towards us. In addition, turbulent internal velocities, of hundreds of $\mathrm{km} \mathrm{s}^{-1}$, are seen in the filaments, as well as gradients of $\gtrsim 200 \mathrm{~km} \mathrm{~s}^{-1}$ over only few hundred parsecs. Although no spectroscopy of fainter parts of the FUV/H $\alpha$ has been published, Sharp (2013, private communication) reports that the line-emitting gas just outside the Outer Filament is largely constrained to tight filaments in space and tight ranges in velocity.

2 The V-shaped Outer Filament can be seen in the lower right of the FUV and H $\alpha$ images in Figure 4 the Inner Filament is closer to the galaxy and not included in Figure 4 but can be seen in Figures 1,2 and 5
We are not aware of any discussion in the literature on the origin of these emission-lines' high velocities in NGC 5128. However, we note that clouds in several starburst galaxies (e.g., NGC 253, NGC 839, M82) have similar velocities. In those objects the clouds are thought to be carried out by a starburst-driven hot wind.

Likely Composition - The diffuse, twisted ribbon appears nearly identical when seen in $\mathrm{H} \alpha$ and FUV; it is also visible in a very deep GALEX Near-UV image (Auld et al. 2012). It is probably a combination of warm lineemitting gas (e.g., Morganti et al.1991 or Sutherland et al. 1993) and very recent star formation (recent enough that some of the newly formed stars still maintain HII regions, e.g., Graham 1998, Mould et al. 2000, Crockett et al. 2012). Our current data do not allow us to discriminate between the two possibilities, but it seems likely that both processes are involved.

The Inner and Outer Filaments are known to contain massive stars, some of them very young ( $\lesssim 4 \mathrm{Myr}$ ), and many of them exciting local HII regions (Graham 1998, Mould et al. 2000, Graham \& Fassett 2002, Rejkuba et al. 2002, Crockett et al. 2011). Our H $\alpha$ and FUV images of the outer ribbon show compact bright spots intermixed with the more diffuse ribbon. Some of these spots can be identified in both images; this suggests they are HII regions around very young stars, embedded in an extended warm ISM. Some FUV-bright regions do not have $\mathrm{H} \alpha$ counterparts (e.g., the extended west arm of the Vshaped Outer Filament). 

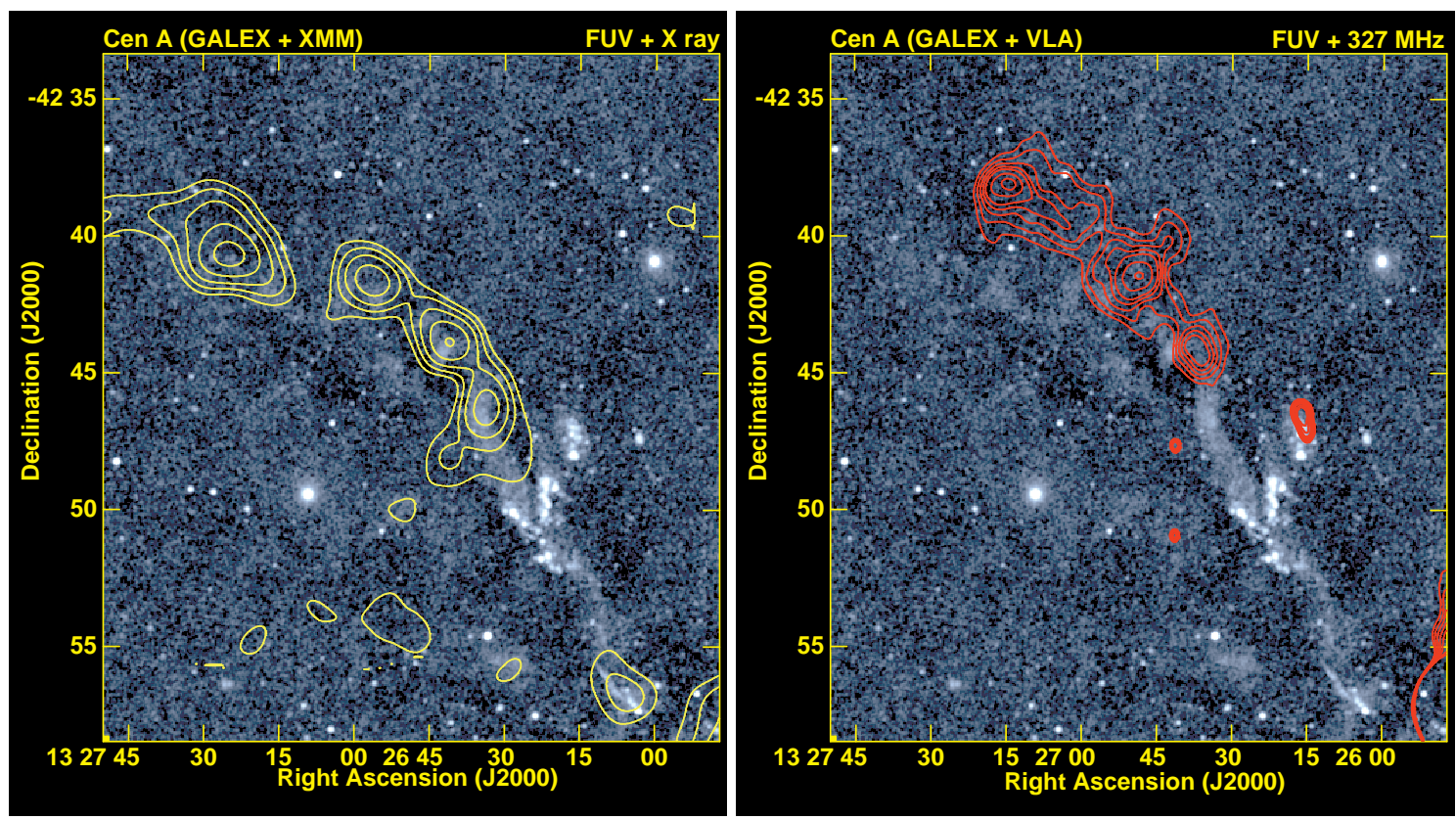

FIG. 5.- Left: Relationship between the X-ray knots (yellow contours; from K09; smoothed XMM-Newton data kindly provided by R. Kraft) and the FUV ribbon (background image, our full-field GALEX image, from figure 1). The X-ray knots begin just north of the V-shaped Outer Filament, and approximately track the center of the twisting FUV ribbon. Right: Relationship between the radio knots (red contours) and diffuse FUV emission (background image). The three radio knots appear to be associated with bends in the diffuse FUV ribbon (see also Figure 4); note that no compact radio emission is associated with the Inner and Outer optical filaments. (The three very compact sources $\mathrm{S}$ and $\overrightarrow{\mathrm{W}}$ of the radio knots are background sources). Radio contours are from a $327 \mathrm{MHz}$ image in Paper 1 , with a beam size $28^{\prime \prime} \times 17^{\prime \prime}$. Contour levels are at 130, 140, 150, 160, 170, 190, $210 \mathrm{mJy} /$ beam.

FUV and $\mathrm{H} \alpha$ emission may arise from diffuse clouds of warm gas; the close structural agreement of the FUV and $\mathrm{H} \alpha$ ribbons suggests cospatial extended emission. Part of the ribbon may be composed of multiphase ISM structures, containing $10^{4} \mathrm{~K}$ gas - emitting $\mathrm{H} \alpha$, [NII], and [O III] lines - and possibly $10^{5} \mathrm{~K}$ gas, radiating in [CIV] at $1549 \AA$ (e.g., Werner et al. 2013, Sparks et al. , 2004). High sensitivity Far-UV spectroscopic observations could test this suggestion.

Some of the ionized gas cannot be excited by young stars alone. The well-studied Inner and Outer Filament gas is characterized by a very high ionization level (Graham \& Price 1981, Morganti et al. 1991). ${ }^{3}$ Young stars cannot account for this, because they reach at most $\sim 15,000 \mathrm{~K}$, while some of the ionized gas requires temperatures of $\sim 10^{5} \mathrm{~K}$.

There is disagreement in the literature about how the gas in the Inner and Outer Filaments has been ionized. Possibilities include photoionization from the AGN (Morganti et al. 1992), flow-induced shocks from a buoyant bubble (Saxton et al. 2001), or shock-produced X-ray photoionization driven by flow of a possible "large-scale jet" (Sutherland et al. 1993). We note that many of the ionizing effects attributed to a nearby jet could equally well be caused by a more diffuse fast flow, such as a galactic wind.

${ }^{3}$ Sharp (2013, private communication) notes that there is a noteable ionization gradient in part of the ribbon, beyond the Outer Filament, but that it will require detailed modelling to interpret.

\subsection{Hot plasma: Radio and X-ray Emission}

The optical/FUV ribbon is closely related to the knotty ridge, seen in radio and X-rays, which sits near the SE edge of the North Middle Lobe. We showed in Paper 1 that the east side of the NML contains three distinct radio knots, each 1.3-1.5 kpc in size, connected by a radio-bright ridge which extends another $\sim 10 \mathrm{kpc}$ inwards toward the galaxy from the knots. A similar, nearly coincident, structure is seen in soft X-rays (Kraft et al., 2009). In paper 1 we noted three possibile explanations for the radio and X-ray knots. (1) They might be extant clouds of cold gas which have been energized by local plasma flow. (2) They might come from interfaces between cooler emission-line clouds and a hot surrounding medium. (3) They might be sites of ongoing star formation; their radio and X-ray power suggests a rate $\sim 0.1 M_{\odot} \mathrm{yr}^{-1}$.

In Figure 5 we show a side-by-side comparison of the X-ray and radio knots, both overlaid on the FUV field. This makes it clear that the knot/bridge structures seen in radio and in X-rays are closely related to each other and also to the $\mathrm{FUV} / \mathrm{H} \alpha$ ribbon.

In the left panel of Figure 5 we show that the X-ray emission knots lie in close correspondence with the ribbon of FUV emission. The X-ray ridge tends to track the center of the FUV ribbon, and both systems show a similar southwards bend at the outer end of the ribbon. The $\mathrm{X}$-ray knots coincide, within the limits of the XMM resolution, with bright regions of the $\mathrm{FUV} / \mathrm{H} \alpha$ ribbon. This correspondence of X-ray, FUV and $\mathrm{H} \alpha$ emission also ex- 
ists in the Inner Filament, where Evans \& Koratkar (2004) detected X-rays, as shown in the lower right corner of the left panel of Figure 5 .

In the right panel of Figure 5 we compare the radio knots to the FUV emission. We find that the radio ridge does not correspond as closely to the ribbon as the X-ray ridge does. The radio ridge/knots are slightly offset to the NW, and the three radio knots actually fall in between the bright regions of the FUV/H $\alpha$ filament. This indicates that whatever excites the radio knots is related to, but not spatially coincident with, the warm ISM filaments seen in optical, FUV and soft X-rays.

Interestingly, Figure 5 shows that both the radio and $\mathrm{X}$-ray knots begin outside the V-shaped Outer Filament. No soft X-ray emission is seen either from that structure, or from the $\mathrm{FUV} / \mathrm{H} \alpha$ ribbon between the Inner Filament and the Outer Filament. The inner part of the radio ridge (galaxy-ward of the knots, not shown here) sits close to, but not obviously associated with, the left arm of the "V" of the Outer Filament. Although X-ray emission is associated with the Inner Filament, radio emission is not.

\subsection{Cold gas: origin of the weather system?}

The origin of the warm gas in the weather system is not well understood. The gas may be initially cold gas which has somehow been injected into the system, or it may be thermally unstable and cooling from the hotter ambient ISM. The first alternative is especially attractive for Cen A, due to the presence of a broken ring of cold gas and dust clouds which orbit the galaxy at radii of $\lesssim 17 \mathrm{kpc}$ (Schiminovich et al. 1994, Charmandaris et al. 2000, Auld et al., 2012). Figure 6 shows the relationship between the FUV ribbon and the HI clouds. The cold material is thought to be left over from a merger with a large gasrich disk 250-750 Myr ago; the same event provided the material that now forms the iconic central dust lane (e.g., Sparke et al. 1996, Quillen et al. 2006, Struve et al. 2010).

One of the clouds making up the ring - which we call the "17-kpc cloud" (labelled in Figure 6) - is of particular interest. Several authors (e.g., Graham 1998, Morganti et al. 1999) have noted that the eastern edge of this cloud appears to be spatially coincident with the western arm of the V-shaped Outer Filament, and have suggested that cold gas ablated from the HI cloud is the source of that filament. Osterloo \& Morganti (2005) showed that the east edge of this cloud - where it is in apparent contact with the $\mathrm{H} \alpha$ and FUV ribbon - has anomalous velocities which agree well with velocities of the turbulent warm gas observed in the adjacent Outer Filament. Furthermore, part of the $\mathrm{FUV} / \mathrm{H} \alpha$ ribbon has a velocity gradient consistent with that of the HI ring (Sharp, 2013, private communication). Both of these observations support the the idea that the cold cloud is a likely source of some or all of the "downstream" ionized gas.

FUV and $\mathrm{H} \alpha$ emission is seen closer to the galaxy center than the 17-kpc HI cloud and the rest of the detected HI system, but no likely cold gas reservoir is apparent for these regions. What might be the source of that warm

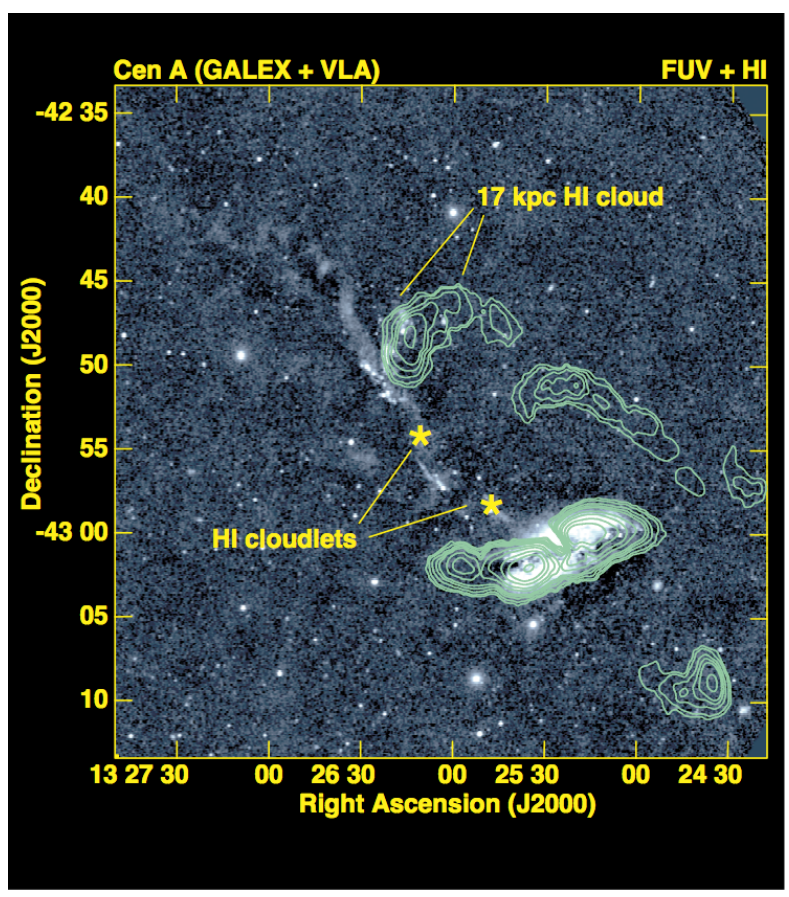

FIG. 6.- Overlay of HI contours superposed on our FUV GALEX image. HI data was obtained with the VLA in D array, image kindly provided by D. Shiminovich. The HI velocities are consistent with a polar ring of cold gas, rotating with the central HI disk. The eastern edge of one of the northernmost HI cloud (labelled as "the 17-kpc cloud") appears to abut the FUV ribbon, suggesting that it may provide some of the raw material forming the warmer ribbon. Two smaller HI clouds, discovered by Struve et al. (2010) and marked here by asterisks, appear to sit right on top of the inner line of FUV emission.

gas? Struve et al. (2010) recently detected two small HI cloudlets, both located along the line of ionized gas that forms the inner part of the weather ribbon (indicated in Figure 6). One of the cloudlets sits near where the inner jet destabilizes and flares out into the North Inner Lobe ( $\sim 5 \mathrm{kpc}$ from the nucleus), and the other sits just outside of the Inner Filament ( $11 \mathrm{kpc}$ from the nucleus). The velocities of the two new cloudlets are somewhat less than the systemic velocity, but they are not as blue-shifted as the emission line gas in the Inner Filament. These cloudlets may be the last remnants of a previous extension to the HI ring, most of which has already been transformed into the inner parts of the $\mathrm{FUV} / \mathrm{H} \alpha$ ribbon through interaction with an outflow.

\subsection{Much of the Weather is Evanescent}

Like terrestrial weather, the most striking features of Cen A's weather system are short-lived. The outer parts of the ribbon cannot have survived the transit out from the parental 17-kpc HI cloud, let alone from the inner galaxy, and they certainly cannot be remnants of a historical merger that happened a few hundred Myr ago. These weather phenomena must have been recently stimulated, by some local driver close to where we see them today. In this section, we explore the range of short timescale constraints acting in the North Transition Region. Because the range of various timescales relevant to the history of 
TABLE 2

Timescales Relevant to the Weather System in Cen A / NGC 5128,

\begin{tabular}{|c|c|c|c|}
\hline Region & Timescale & Reference & Comments \\
\hline \multicolumn{4}{|l|}{ Inner galaxy: } \\
\hline dynamic age of inner lobes & $\sim(1-2) \mathrm{Myr}$ & 1.1 & shock around SIL \\
\hline recent star formation & $2-50 \mathrm{Myr}$ & 4 & youngest stars in disk \\
\hline age of starburst & $\gtrsim 50 \mathrm{Myr}$ & 8 & oldest blue stars in disk \\
\hline \multicolumn{4}{|l|}{ Weather system } \\
\hline ages of young Stars & 4-15 Myr & 3.4 & stellar colors/isochrones \\
\hline cloud radiative cooling time & $\sim 15 \mathrm{yr}$ & $\overline{3.4}$ & for $10^{4} \mathrm{~K}$ emission-line cloud \\
\hline emission-line cloud expansion time & $\lesssim 0.6 \mathrm{Myr}$ & 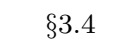 & internal cloud pressure, unconfined \\
\hline emission-line filament dispersal time & $\lesssim 10-15 \mathrm{Myr}$ & 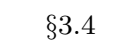 & cloud velocity dispersion \\
\hline radio/X-ray knot expansion time & $\sim 0.5-3 \mathrm{Myr}$ & 3 & if overpressure gas clouds \\
\hline age of radio/X-ray knots & $\sim 10 \mathrm{Myr}$ & $\overline{3.4}$ & if star-forming regions \\
\hline Kelvin-Helmholtz cloud shredding time & $1-2 \mathrm{Myr}$ & $\bar{A} .1$ & from linear growth rate \\
\hline cloud evaporation by thermal conduction & $\gtrsim 2 \mathrm{Myr}$ & A.1 & Spitzer conductivity \\
\hline transit time to end of ribbon, for filaments & $50-85 \mathrm{Myr}$ & 8 & from observed cloud velocities \\
\hline \multicolumn{4}{|l|}{ North Middle Lobe: } \\
\hline wind transit time through NML & $10-30 \mathrm{Myr}$ & 5.3 & assumes $2000-6000 \mathrm{~km} \mathrm{~s}^{-1}$ wind \\
\hline diffuse NML & $\lesssim 16 \mathrm{Myr}$ & 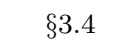 & overpressure expansion if unconfined \\
\hline synchrotron aging & $\lesssim 20 \mathrm{Myr}$ & 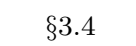 & at/above $90 \mathrm{GHz}$ \\
\hline \multicolumn{4}{|l|}{ Outer radio Lobes: } \\
\hline shortest radiative lifetime & few Myr & 1.1 & for $\gamma$-ray-loud electrons \\
\hline re-energization & $\sim 30 \mathrm{Myr}$ & 1.1 & decay time for internal turbulence \\
\hline dynamic age & $\sim 1 \mathrm{Gyr}$ & 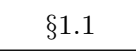 & from dynamic models \\
\hline \multicolumn{4}{|l|}{ Dynamics } \\
\hline time since last major merger & $2-4 \mathrm{Gyr}$ & $\$ 1$ & intermediate age stars in halo \\
\hline time since last (minor) merger/ disk settling time & $250-750 \mathrm{Myr}$ & 3.3 & disk modelling of HI \& IR; "blue arc" \\
\hline rotation time for HI ring & $\lesssim 370 \mathrm{Myr}$ & $\overline{3.3}$ & $\mathrm{~V}_{\text {rot }} \gtrsim 250 \mathrm{~km} \mathrm{~s}^{-1}$ \\
\hline
\end{tabular}

the inner galaxy and/or the weather system is large, we collect those timescales for reference in Table 2. We will show in Section 6 how these timescales can be accomodated in the presence of a galactic-scale wind.

Young Stars - The well studied Inner and Outer Filaments contain very young stars (age $\sim 4-15 \mathrm{Myr}$; Mould et al. 2000; Rejkuba et al. 2002, 2011; Crockett et al. 2011). Some of these (e.g., those in the Inner Filament and the eastern branch of the Outer Filament) have associated HII regions; others (western branch of the Outer Filament) do not. Stars hot enough to ionize HII regions have typical lifetimes $\lesssim 10$ Myr.

The FUV emission places less stringent constraints on stellar ages: we expect to see FUV emission from massive $\mathrm{O}$ stars for $\sim 10 \mathrm{Myr}$ and from B stars for up to $100 \mathrm{Myr}$. Although we have no direct information on the nature of the outer $\mathrm{FUV} / \mathrm{H} \alpha$ ribbon, the same lifetime arguments will apply if the ribbon emission comes from recently formed stars.
Emission-line clouds: existence - Individual emission-line clouds are subject to several disruptive effects. To estimate the associated timescales, which depend on cloud parameters, we note that the relatively large $(\gtrsim 100 \mathrm{pc}$ ) emission-line "clouds" studied in the Inner and Outer Filaments (e.g., Graham \& Price 1981) are actually aggregations of much smaller clouds (Morganti et al. 1991, also the $\mathrm{H} \alpha$ image in the left panel of Figure 4). Following the analysis of Morganti et al. (1991), who studied individual clouds in the Inner Filament and some parts of the V-shaped Outer Filament, we define a "typical" emission line cloud: size $a_{c} \sim 10 \mathrm{pc}$, density $n_{c} \sim 30 \mathrm{~cm}^{-3}$ and temperature $T_{c} \sim 10^{4} \mathrm{~K}$. We assume these numbers are also representative of emission line clouds further out in the weather system.

These clouds cannot last long in their present form, for at least two reasons.

1) A typical emitting cloud requires ongoing energization to offset rapid cooling and recombination. The radiative cooling time is extremely short: estimating the cool- 
ing function as $\Lambda\left(T_{c}\right) \sim 10^{-22} \mathrm{erg} \mathrm{cm}^{3} / \mathrm{s}$ for $T_{c} \gtrsim 10^{4} \mathrm{~K}$, we see $t_{\text {cool }}=k T_{c} / n \Lambda\left(T_{c}\right) \sim 15 \mathrm{yr}$. This is a short enough timescale for changes to to be observable, if one has sufficient angular resolution. For instance, a $10 \mathrm{pc}$ cloud would subtend $\sim 0.5^{\prime \prime}$, so that secular changes in such clouds would be accessible to current telescopes over the time that the filaments have been studied.

2) A typical emission line cloud is at much higher pressure than the surrounding ISM. The cloud has $p_{c}=$ $2 n_{c} k T_{c} \sim 8 \times 10^{-11} \mathrm{dyn} \mathrm{cm}^{-2}-$ significantly higher than the pressure of the local galactic ISM $\left(8.5 \times 10^{-13}\right.$ dyn $\mathrm{cm}^{-2}$, Kraft et al., 2009) If these clouds are not confined, they will expand at their internal sound speed $(\sim 15$ $\left.\mathrm{km} \mathrm{s}^{-1}\right)$; thus a 10-pc cloud will dissipate in only $\sim 0.6$ Myr (see also Morganti et al. 1991).

Emission-line clouds: kinematics - Emission-line clouds within both the Inner and Outer Filaments show high random velocities ( $\sim 150-400 \mathrm{~km} \mathrm{~s}^{-1}$; Graham and Price 1981, Morganti et al. 1991) within small regions $(\sim 100$ pc). These structures will disperse in $\lesssim 10-15 \mathrm{Myr}$ unless they are somehow confined. Less is known about velocities in the weather ribbon beyond the Outer Filament. Graham and Price (1981) measured velocities in a few of the brightest regions of the ribbon, and found velocity dispersions similar to those in the Inner and Outer Filaments.

Radio and X-ray knots - We showed in Paper 1 that if the radio and $\mathrm{X}$-ray knots are diffuse plasma clouds, they are strongly overpressured, $\gtrsim 20 p_{I S M}$. Thus, they will dissipate quickly if they are not confined. The X-ray knots will survive no longer than $\sim 3 \mathrm{Myr}$ (paper 1 , also Kraft et al. 2009), while the radio knots will last no more than $\sim 0.5 \mathrm{Myr}$ (paper 1).

Alternatively, we noted in paper 1 that the the X-ray and radio knots might come from star-forming regions. Hard X-rays, if observed, could be an indicator of shortlived, massive stars with lifetimes $\sim 10$ Myr. Because the region has not been observed in hard X-rays, we cannot definitely say that the knots must be sites of recent star formation; but evidence for such young stars elsewhere in the weather ribbon (e.g., Mould et al. 2000, Crockett et al. 2012) suggests that the X-ray/radio knots could contain similarly young stars if they are star-forming regions.

Transit times from the inner galaxy- The survival timescales for filaments and knots are much shorter than the time it would take for gas from either the $17-\mathrm{kpc} \mathrm{HI}$ cloud or from the inner galaxy to have reached the weather system. The weather ribbon extends $\sim 35 \mathrm{kpc}$ from NGC 5128 , and $\sim 18 \mathrm{kpc}$ from the HI cloud (both distances measured in the sky plane). The optical filaments are moving away from NGC 5128 and towards us at $\sim 350$ $\mathrm{km} \mathrm{s}^{-1}$ (line of sight velocity; Section 5.1). If the cloud speed in the sky plane is comparable, and if the ribbon material originated in the $17-\mathrm{kpc} \mathrm{HI}$ cloud, it would take $\sim 50 \mathrm{Myr}$ for the line-emitting gas to travel from the 17$\mathrm{kpc} \mathrm{HI}$ cloud to the outer end of the ribbon. Alternatively, it would take $\gtrsim 85 \mathrm{Myr}$ for the material in the weather ribbon to travel from the center of the galaxy to its present location.

It follows that any young stars in the outer regions of the weather ribbon must have formed there. They are too young to have been formed close to the galaxy and transported out. Similarly, the emission-line clouds and radio/X-ray knots are unlikely to have lived long enough to survive the journey from the galaxy in their present form. They too must be energized in situ in the weather ribbon.

Other short-lived radio phenomena - In Paper 1 we pointed out three lines of further evidence for recent energy transport through, and dissipation within, the North Transition Region. (1) We found there that the North Middle Lobe is at a higher pressure than the surrounding ISM of the galaxy; if were a static structure, it would expand away in $\lesssim 16$ Myr. (2) We noted that the detection of the North Middle Lobe at $90 \mathrm{GHz}$ (by WMAP; Hardcastle et al. 2009) requires the electrons radiating at those frequencies to have been re-energized no more than $\sim 20$ Myr ago. (3) Because the Outer Lobes must have been re-energized no more than $\sim 30$ Myr ago to keep them shining in $\gamma$-rays as well as radio (Eilek 2014), the necessary energy must have flowed through both Transition Regions at least this recently.

\section{A WIND FROM THE CENTRAL GALAXY}

In the previous section we showed that the diverse phenomena which comprise the weather system in the North Transition Region require in situ driving, because they are too short-lived to have been transported out from NGC 5128. The AGN is a likely energy source, but without a (detected) jet on 10-30 kpc scales it is unclear how the energy can make it from the galactic core to the weather system.

In this section we explore an alternative mechanism: a diffuse galactic wind. The GALEX image shown in Figure 1, also Figure 7, shows that an active starburst is currently underway in the dusty disk of NGC 5128. With this starburst, in addition to the AGN, we have two potential power sources in the inner galaxy. We will argue that the starburst - probably enhanced by energy from the AGN - is driving a wind which can provide the necessary energy transport into and through the Transition Regions.

\subsection{Star formation rate in NGC5128}

FUV radiation reveals young stars not hidden by gas and dust clouds (or their radiation scattered into our line of sight), while FIR radiation traces young stars that are heavily obscured. We therefore combine FUV (1344$1786 \AA)$, FIR $(8-1000 \mu)$, and radio $(90 \mathrm{~cm})$ measurements to determine the strength of the starburst. 


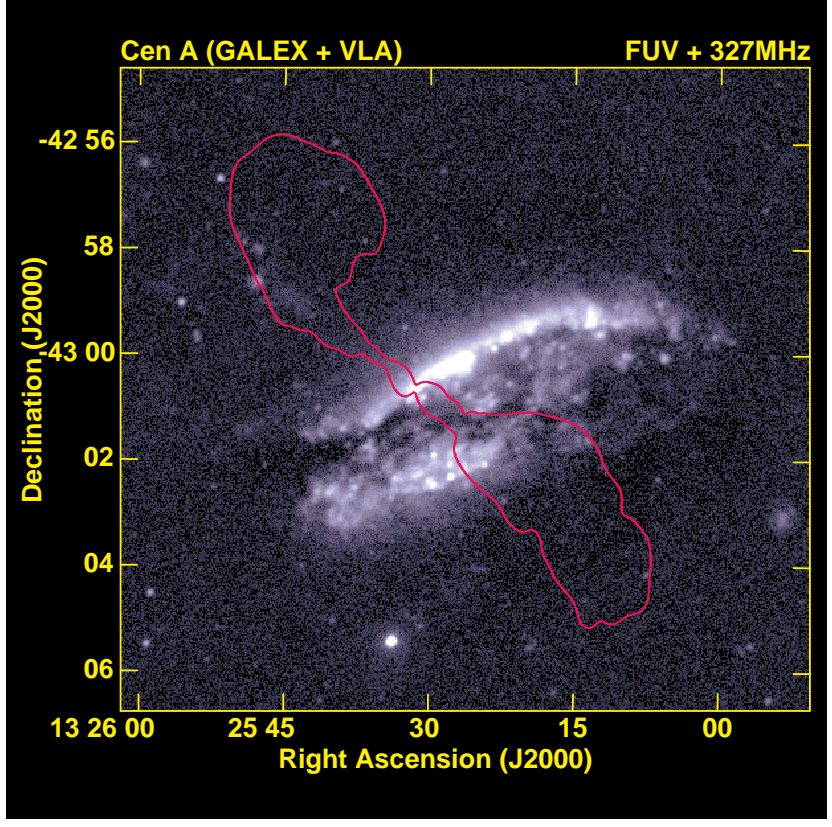

FIG. 7.-FUV GALEX image of the inner $13 \mathrm{kpc}$ of NGC 5128 . The underlying image is an unsmoothed image, with resolution of $4.5^{\prime \prime}$. The red contour is the $1.1 \mathrm{Jy} /$ beam brightness level in our unconvolved 90-cm image of the Inner Lobes (Paper 1). The bright FUV emission from the central gas/dust disk reveals a strong starburst in the central region of the NGC 5128. FUV emission is seen above and below the dust.

Unobscured star formation: Far-UV- We used the FUV image to characterize the unobscured part of the starburst. The burnt-out display in Figure 1 shows that FUV emission can be detected for $\sim 1.5^{\prime}$ above and below the dust disk plane, and for a total distance of $\sim 8^{\prime}$ along the dust plane. The disk is quite thin, and warped multiple times (Quillen et al.1993); Figure 7 shows the detailled structure of the central $13 \mathrm{kpc}$ of the NGC 5128 as seen in FUV.

We used the AIPS program BLSUM to integrate the emission from the central galaxy in a hand-drawn region of size $\sim 7.3^{\prime} \times 2.9^{\prime}(\sim 8.3 \times 3.3 \mathrm{kpc})$, enclosing all of the FUV emission shown in Figure 7 but excluding the base of the inner jet and nearby bright stars. We measured the local background in several nearly star-free boxes near the galaxy (within $10^{\prime}$ ) and subtracted that level from the total measured flux. Integrating across the GALEX bandpass, we derive the observed FUV flux of the central starburst disk, $F_{\mathrm{FUV}}^{\mathrm{obs}} \gtrsim 3.2 \times 10^{-12} \mathrm{erg} \mathrm{s}^{-1}-\mathrm{cm}^{2} / \AA$. Multiplying by the effective GALEX bandwidth ( $268 \AA$ for FUV, Morrissey et al. 2007), and taking a 3.8-Mpc distance, we estimate the directly observed FUV luminosity ${ }^{4}$ as $L_{\mathrm{FUV}}^{\mathrm{obs}} \sim 1.5 \times 10^{42} \mathrm{erg} \mathrm{s}^{-1}$.

To correct for foreground Galactic extinction, we use $A_{\mathrm{FUV}}=0.91 \mathrm{mag}$ (Gil de Paz et al., 2007). Thus, the unobscured FUV luminosity from the galaxy is boosted by a factor 2.3 , to $L_{\mathrm{FUV}}^{\text {corr }} \sim 3.5 \times 10^{42} \mathrm{erg} \mathrm{s}^{-1}$. We convert this to a star formation rate (SFR) with the proxy relation

4 Throughout this paper, $\mathrm{L}_{\mathrm{FUV}}$ refers to the luminosity in the GALEX FUV bandpass, $\lambda 1344-1786 \AA$.
$\mathrm{SFR}=4.4 \times 10^{-44} L_{\mathrm{FUV}}$ (Murphy et al. 2011 ; units of SFR are $M_{\odot} /$ yr and units of $L_{\mathrm{FUV}}$ are erg s ${ }^{-1}$ in $G A L E X$ FUV bandpass). We thus estimate the unobscured star formation rate in the disk of NGC 5128: (SFR) naked $\sim$ $.15 M_{\odot} / \mathrm{yr}$. If UV light escapes preferentially above and below the disk, and is not scattered into our line of sight, $(\mathrm{SFR})_{\text {naked }}$ may be higher.

Obscured star formation: Far-IR - The strength of an obscured starburst is commonly determined from its bolometric IR luminosity $\left(L_{\mathrm{FIR}}: 8-1000 \mu\right)$. To estimate $L_{\text {FIR }}$ for NGC5128, we started with its IRAS fluxes, $\sim 217 \mathrm{Jy}$ at $60 \mu$, and $\sim 501 \mathrm{Jy}$ at $100 \mu$ (Rice et al. 1988). We converted these to $40-120 \mu$ flux, using FIR $=$ $1.26 \times 10^{-14}\left[2.58 S_{60 \mu}+S_{100 \mu}\right]$ (e.g, Helou et al. 1985). We then multiplied (FIR) by the $\sim 1.5$ conversion factor typical for FIR-selected galaxies (Yun et al. 2001) finding that the bolometric flux $L_{\text {FIR }} \sim 9.3 \times 10^{9} L_{\odot} / \mathrm{yr}$.

Both Kennicutt (1998) and Murphy et al. (2011) give proxy relations to convert $L_{\text {FIR }}$ to a star formation rate (SFR), with small differences reflecting different assumptions detailed conditions within the starburst. Kennicutt gives $\mathrm{SFR} \sim 1.7 \times 10^{-10} L_{\mathrm{FIR}} ;$ Murphy et al. give $\mathrm{SFR} \sim 1.47 \times 10^{-10} L_{\mathrm{FIR}}$; here, SFR is measured in $\left(M_{\odot} / \mathrm{yr}\right)$ and $L_{\mathrm{FIR}}$ is measured in solar luminosities. Taking a rough mean of these, we estimate the rate of obscured star formation in NGC5128 as $(\mathrm{SFR})_{\text {obsc }} \sim 1.6 M_{\odot} \mathrm{yr}^{-1}$

Total star formation: Radio - We also used our 90-cm radio image (Paper 1) as an independent check of the star formation rate in the central gas/dust disk. Because radio luminosity does not suffer from extinction, it should give the total star formation rate (obscured plus unobscured). We pointed out in Paper 1 that we detect a "ruff" of radio emission, transverse to the Inner Lobes and approximately coincident with the gas/dust disk (compare, e.g., Figure 1 or 6 of paper 1 to Figure 7 of this paper). We used the flux of this ruff as a separate proxy for the star formation rate, as follows. We measured the mean flux density of the NW and SE regions of the disk (outside of the bright central AGN) as $165 \mathrm{mJy} /$ beam, which we estimate includes a background of $25 \mathrm{mJy} /$ beam. We estimated the total disk area as $5.3^{\prime} \times 1.5^{\prime}$; thus the total radio flux from the disk $\sim(140 \mathrm{mJy} /$ beam $) \times(56$ beams $) \simeq 7.84$ Jy at 327 $\mathrm{MHz}$. Because of the uncertainties in estimating the radio background and interpolating across the AGN, this flux estimate is probably only good to a factor $\sim 2$.

We scaled this flux estimate to $1 \mathrm{GHz}$ using the mean spectrum of star-forming galaxies in this frequency range, $S(\nu) \propto \nu^{-0.55}$ (Marvil et al. 2014). This gives a $1-\mathrm{GHz}$ radio luminosity $L_{1 \mathrm{GHz}} \sim 7 \times 10^{28} \mathrm{erg} \mathrm{s}^{-1} \mathrm{~Hz}^{-1}$ for the star-forming disk in Cen A. We then used the proxy relation from equation (16) of Murphy etal (2011), which combines thermal and non-thermal radio emission to estimate a total rate as $\mathrm{SFR}=5.8 \times 10^{-29} L_{1 \mathrm{GHz}}$. In this relation, the units of SFR are $M_{\odot} / \mathrm{yr}$, the units of $L_{1 \mathrm{GHz}}$ are erg $\mathrm{s}^{-1} \mathrm{~Hz}^{-1}$; we have assumed $T=10^{4} \mathrm{~K}$ and $\nu=1$ $\mathrm{GHz}$. From this we estimate the radio-derived SFR in the 
disk of Cen $\mathrm{A}$ as $\sim 4 M_{\odot} / \mathrm{yr}$. Although this rate is formally larger than the net SFR we derive from FUV and $\mathrm{FIR}, \sim 1.75 M_{\odot} / \mathrm{yr}$, we do not take the difference seriously because of the large uncertainties in our measurement of the radio flux. We thus argue that the radio data support the FUV+FIR data, showing that the central disk in NGC 5128 is currently forming stars at a rate $\sim 2 M_{\odot} / \mathrm{yr}$.

\subsection{The central starburst and its wind}

Combining the obscured and unobscured star formation rates, and comparing those results to our radio-derived estimates of the total star formation rate, we estimate the central disk in NGC 5128 hosts a starburst with total SFR $\sim 2 M_{\odot} \mathrm{yr}^{-1}-$ comparable to that estimated for the Milky Way, but concentrated in a region only a few kpc across.

To estimate the age of starburst, we ideally need a good census of the young and middle-aged stars in the region. However, the disk in NGC5128 has not been the subject stellar population studies, probably because it is such a crowded, obscured region. A few studies of blue stars and accompanying $\mathrm{H} \alpha$ emission along the edges of the disk (Dufour et al. 1979, Bland et al. 1987, Minitti et al. 2004) have revealed individual blue stars or clusters with ages from 2-50 Myr. This places a lower limit on the age of the starburst, and shows that it is still active today. To place an upper bound on the starburst age, we note that starbursts in other galaxies are typically estimated to last up to several hundred Myr (e.g., Crnojevic et al. 2011, McQuinn et al. 2009, 2010). It therefore seems likely that the starburst in NGC5128 has been going on for at least $\sim 50-100$ Myr.

As with starbursts in other galaxies, we expect the starburst in the disk of NGC5128 to drive out a wind. To estimate the strength of the wind we use the range of starburst models of Strickland \& Heckman (2009), for an intermediate age starburst (20-50 Myr), scaled to $\lesssim 2 M_{\odot} /$ yr. From this we estimate the total wind power, $P_{w} \sim 1 \times 10^{42} \mathrm{erg} \mathrm{s}^{-1}$, and total mass flux $\dot{M}_{w} \sim$ $0.5 M_{\odot} \mathrm{yr}^{-1}$ (to north and south of the disk; following Strickland \& Heckman 2009). Thus, a pure-starburst wind would contribute only a few percent of the power estimated to come from the AGN (as summarized in Table 3 , using estimates from Paper 1).

Can we be certain this starburst drives out a wind? There is a general consensus (e.g., Strickland 2009, Lehnert \& Heckman 1996) that a star formation rate $\gtrsim 0.1 M_{\odot} / \mathrm{yr}-\mathrm{kpc}^{2}$ is sufficient to overcome the inertia of cold ISM in the starburst region and drive out a wind. While we do not know the internal structure of the starburst in NGC 5128 - it is obscured by the disk - infrared imaging (Parkin et al. 2012, using Herschel; also Rice et al.1988, from IRAS) suggests it is concentrated in the inner $\sim 5 \mathrm{kpc}$ of the disk. If we assume the $2 M_{\odot} \mathrm{yr}^{-1}$ starburst is uniformly distributed over a disk $5 \mathrm{kpc}$ in diameter, we derive an areal star formation rate $\sim 0.1 M_{\odot} \mathrm{yr}^{-1} \mathrm{kpc}^{-2}$. As this is just at the nominal (emperical) threshold for wind driving, we argue that the star- burst in the core of NGC 5128 is capable of driving out a wind and is likely to do so, albeit perhaps not as strong as those in better known systems (e.g., M82 or NGC 1569).

TABLE 3

Power estimates for Cen A

\begin{tabular}{ccc}
\hline Region / Epoch & $\begin{array}{c}\text { Power }^{a} \\
\left(\mathrm{erg} \mathrm{s}^{-1}\right)\end{array}$ & Timescale \\
\hline Jets, present-day & \\
Inner lobes, over lifetime & $\sim(2-6) \times 10^{43}$ & $\sim(1-2) \mathrm{Myr}$ \\
Starburst wind, recently $^{c}$ & $\sim 1 \times 10^{42}$ & $\gtrsim 50 \mathrm{Myr}$ \\
Outer lobes, over lifetime $^{2} \gtrsim(2-5) \times 10^{43}$ & $\sim 1 \mathrm{Gyr}$ \\
\hline
\end{tabular}

a The total power to both sides of the galaxy assuming symmetric north and south outflows. Radio-based estimates taken from Neff et al. (2014, Paper 1).

${ }^{b}$ Assuming jets uniformly filled with electron-positron plasma.

${ }^{c}$ Assumes the wind is driven by $\mathrm{a} \sim 2 M_{\odot} \mathrm{yr}^{-1}$ starburst, and is not augmented by the AGN (Section 4.2 this paper).

\subsection{Does the AGN augment the wind?}

At the present epoch, Cen A contains both an ongoing starburst and a recently recollimated (or perhaps restarted) AGN. In the previous section we described a stand-alone wind, i.e., one driven only by the starburst. However, this is likely to be too simple. We expect that relativistic outflows from the AGN will interact with, and perhaps energize, the wind. We do not know the recent history of the AGN (mostly active? mostly quiet?), but we can bound the problem by two possibilities.

A long silence from the $A G N-$ As we discuss in Paper 1 , the inner radio source is $\lesssim 2 \mathrm{Myr}$ old (Croston et al. 2009). One possibility is that the AGN has been dormant for much or all of the duration of the starburst. This could be causal, perhaps because supernovae-driven turbulence within the starburst disk disrupts the accretion flow, and/or removes substantial amounts of material that would otherwise have accreted onto the central black hole (e.g., Wild et al. 2010, Davies et al. 2007).

To be specific, we assume the AGN in NGC5128 has been quiet for $\sim 50-100 \mathrm{Myr}$ (the likely duration of the central starburst, also the delay that Davies et al. (2007) suggest between start of a starburst and the restarted feeding of the AGN). If this is the case, any radio-loud plasma from previous active episodes of the AGN would long ago have reached and/or dispersed within the Outer Lobes. For instance, if the disconnected Inner Lobe plasma kept a coasting speed $\sim 3000 \mathrm{~km} \mathrm{~s}^{-1}$ (comparable to the deprojected advance speed of the Inner Lobes; $c f$. paper 1), it would reach $150 \mathrm{kpc}$ in $50 \mathrm{Myr}$ - well beyond the Transition Regions discussed here. In this case, energy and mass flow within the Transition Regions will be limited to that of the basic, starburst-driven wind described above.

While we can't prove or disprove this picture, we find 
it unlikely for several reasons. It would require us to be catching the AGN at a special time, only 1-2 Myr since its restart after a long silence. In addition, we argue below (in Sections 6.2 and 6.4 that a wind driven only by the starburst does not have enough power to support the emission line clouds in the weather system, or the radio emission from the North Middle Lobe. Finally, essentially every double radio galaxy known hosts a currently active, radio-loud AGN. Even the rare radio galaxies without detected jets connecting the AGN to the radio lobes have detectable jets on kpc scales (e.g., Fornax A, Fomalont et al. 1989; 3C310, van Breugel \& Fomalont 1984). If it were common for the AGN supporting a large radio galaxy to go dormant for $\sim 10 \%$ of the age of the source, we would expect to see a similar fraction of the radio galaxy population with no radio core - which is not the case.

An AGN coexisting with the starburst - An alternative possibility is that the AGN, recently restarted, has only been quiet for a time short compared to the duration of the starburst. Perhaps the AGN "burbles along", dying down and then reactivating every few Myr. This may happen, for instance, if most of the starburst is sufficiently spread out within the inner few $\mathrm{kpc}$ of the star-forming disk that it has little effect on the accretion flow close to the AGN. As we discussed in paper 1 , as the restarted jets propagate into the wind, different evolution paths are possible when they grow to tens of kpc scales. They may continue to be identifiable as jets, without much effect on the ambient wind flow. In this case, we might expect "jet fragments" to continue to coast within the ambient wind when the AGN again becomes quiet. Alternatively, the jets may turn into more diffuse, turbulent plumes, with the potential to impact and energize the local wind flow. In this case we might expect the jet plasma to lose its identity as a jet, and to mix with the ambient wind.

As an example, assume the AGN has been dormant for only $\sim 5$ Myr. If this is the case, the jet plasma from the previous cycle would still be within the Transition Regions $\left(3000 \mathrm{~km} \mathrm{~s}^{-1} \times 5 \mathrm{Myr}=15 \mathrm{kpc} ; 3000 \mathrm{~km} \mathrm{~s}^{-1}\right.$ is the estimated advance speed of the Inner Lobes, Paper 1), and would still be radio-loud (estimating the synchrotron lifetime at several tens of Myr; e.g., paper 1). Because we don't see any such jet remnants within the Transition Regions in Cen A, we speculate that jets from the previous cycle must have disrupted, dispersed and mixed with the ambient wind plasma. It follows that the mass and energy from the from AGN may have been deposited locally in the wind - perhaps quite close to the base of the wind and thus became part of the diffuse wind flow.

\subsection{Are there alternative drivers for the weather?}

Given that the observational evidence for a wind is only modest, and that the power of an unaugmented wind is weak compared to the AGN power, one might ask if some other mechanism can drive weather system.

Photoionization - Morganti et al. (1991, 1992), suggested that the Inner and Outer Filaments are photoionized by beamed radiation from a blazar. However, Hardcastle et al. (2003) suggest that the radio and X-ray brightness ratios in the inner jet appear inconsistent with a misdirected blazar model. Sutherland et al. (1993) point out that the ionization gradient noted by Morganti et al. (1992) does not point towards the nucleus, as might be expected if the Inner Filament were ionized by beamed EUV-Xray nuclear radiation. Together with Viegas \& Prieto (1992), Sutherland et al. (1993) also mention difficulties reproducing the observed $[\mathrm{OIII}] I_{4363} /\left(I_{5007}+I_{4959}\right)$ ratio using pure photoionization models. Crockett et al. (2012) note that a beamed photoionization model also fails to account for the highest excitation lines (e.g., Graham 1998, Evans and Koratkar 2004). Furthermore, ionization by beamed radiation, by itself, does not explain the complex velocity field of the ionized gas in the Filaments.

Relative orientations also do not support the idea of beamed photoionization. The inner jet is oriented at an an angle of $30-41^{\circ}$ (depending on distance from the nucleus), with an opening angle of $\sim 8-10^{\circ}$. The Inner Filament is roughly aligned with the inner jet, with an orientation of $\sim 28-33^{\circ}$ degrees, and thus it might might plausibly be ionized by beamed radiation from the nucleus or inner jet. However, the jet is not aligned with the Outer Filament (orientation $\sim 44-53^{\circ}$ ), or with most of the outer ribbon structure (orientation $\sim 40-56^{\circ}$ ).

Another possibility is that the FUV and emission-line regions are illuminated by the star-forming disk. However, the agreement between radio-determined star formation rates and UV/IR-determined star formation rates (Section 4) precludes the possibility of significant amounts of hidden Far-UV emission being emitted along the dustdisk's axis.

Are filaments in cooling cores a similar phenomenon? - Filaments of emission-line gas and young stars are also found close to central radio galaxies in cooling-core clusters (e.g., Fabian 2012; Canning et al. 2014). These filaments are similar to the weather system in Cen A: they need ongoing energization, and they sit in a dynamic, hot $\left(T \sim 10^{7} \mathrm{~K}\right)$ ambient medium. What can we learn from them? Although the filaments in cooling-core clusters sit in a denser ambient medium than that which surrounds the weather system in Cen A, there are interesting similarities. The thermal state of filaments in cooling cores is far from understood, but there are several possibilities. They may be energized by energy transfer from the surrounding medium (e.g., heat conduction, Sparks et al. 2004, or cosmic ray penetration, e.g. Fabian 2012). The filaments may also be energized dynamically, either by infall of the residual cooling flow, or by expansion work done by the radio galaxy if the filaments happen to sit close to the radio lobe edges.

The cooling-core analogy is not perfect, however. The energetics of cooling cores are ultimately driven by a combination of slow cooling inflow and radio lobe expansion. Neither of these are clearly available for the Cen A weather system. There is no sign of cooling cores in 
small galaxy groups such as that which hosts NGC 5128. The radio-loud plasma in both Transition Regions is more likely to expand, or flow, into the tenuous channels provided by the pre-existing Outer Lobes. Thus, a different driver is needed for the weather system; the wind we suggest is flowing through both Transition Regions can provide that driver. If such a wind exists, the mechanisms by which energy is transferred to the cool gas clouds may be quite similar in Cen A and in cooling cores. We discuss wind energization of the weather system in Cen A in more detail in section 6 .

\section{A WIND THROUGH THE TRANSITION REGIONS}

In the previous sections we have shown that many of the "weather"-related phenomena in the North Transition Region are short-lived, and require ongoing energization - even though there seems to be no AGN-driven jet in the region at present. We have also suggested that the starburst in the central gas disk, perhaps aided by power from the AGN, is driving out a strong galactic wind. We speculate that this wind can play the role of the (undetected) jet: effects attributed to jet-cloud interactions can equally well be caused by wind-cloud interactions. In this section we explore simple models of such a wind. Although there is no robust detection of a hot wind in NGC 5128, we can take advantage of what is known about the winds in some well-known starburst galaxies (e.g., M82, NGC 253, NGC 2146) to understand the wind in NGC 5128.

\subsection{Envisioning the wind}

Basic models of starburst winds (e.g., Chevalier \& Clegg 1985, Strickland \& Heckman 2009) show that the wind starts slowly, close to the starburst, but quickly accelerates and becomes asymptotically supersonic. The key property of the wind is that its terminal speed is a few times the effective sound speed (governed by specific internal energy, both thermal and nonthermal) in its core. Detailed calculations for a supernova-driven starburst wind (e.g., Strickland and Heckman, 2009) find $T_{I S M} \sim 10^{8} \mathrm{~K}$ - assuming the starburst energy is efficiently thermalized and that there is not too much cold material in the nearby ISM. For numerical estimates, we assume such a wind has been going for $\sim 50-100 \mathrm{Myr}$, at power $P_{w} \sim 1.0 \times 10^{42} \mathrm{erg} \mathrm{s}^{-1}$, with mass flux $\dot{M}_{w} \sim 0.5 M_{\odot} \mathrm{yr}^{-1}$. We conservatively ${ }^{5}$ estimate the asymptotic wind velocity as $v_{w} \sim 2000 \mathrm{~km} \mathrm{~s}^{-1}$.

If the starburst wind has been enhanced by relativistic plasma from the AGN, we expect a hotter, faster wind. For a specific example, let the AGN deposit $\sim 1 \times 10^{43}$ erg $\mathrm{s}^{-1}$ in the plasma near the base of the wind (in the range of the likely total AGN power, $c f$. Table 3). Because the AGN outflow carries very little mass, we assume the enhanced wind still has mass flux $\dot{M}_{w} \sim 0.5 M_{\odot} \mathrm{yr}^{-1}$, as estimated for the non-enhanced wind. Because $P_{w} \propto v^{2}$

\footnotetext{
5 This assumes the wind is not totally "cold", but retains $\sim 1 / 3$ of its power in advected internal energy.
}

at constant $\dot{M}_{w}$, we estimate the wind speed as $v_{w} \sim 6300$ $\mathrm{km} \mathrm{s}^{-1}$ in the AGN-boosted wind.

We note that these high wind speeds do not describe emission-line clouds in the wind. Both observations (e.g., Forster-Schreiber et al. 2001) and modelling (Cooper et al. 2008, 2009) show the starbursting disk is inhomogeneous, containing distributed star-forming clusters, each of which drives out its own wind. The individual winds eventually merge into a large-scale, diffuse starburst wind, which entrains and accelerates streams and filaments of cooler, denser gas (from the starburst disk or from interfaces with the local ISM). The entrained dense clouds do not attain the full speed of the hot wind, but they do reach speeds of a few to several hundred $\mathrm{km} \mathrm{s}^{-1}$, becoming the emission-line filaments which characterize winds in well-studied starburst galaxies (e.g., NGC 253, Westmoquette et al.2011; NGC 839, Rich et al. 2010). The similarity of the cloud outflow speeds in those galaxies to the speeds of the emission-line clouds in the Cen A weather system (Section 3.1) suggests that a similar wind may blow through the North Transition Region in Cen A.

\subsection{Observational evidence for a wind}

Is there any observational evidence of a galactic wind in NGC 5128? Winds are easily identified in edge-on disk galaxies by bipolar streams, extending several kpc above and below the galaxy, detected in optical (emissionline gas) and infrared (dust and cooler gas) (e.g., M82, NGC3079, NGC1482, NGC 253; Veilleux et al.2005). Haloes associated with these winds can be detected at radio wavelengths, either as molecular lines from cold gas, (e.g., Sugai et al. 2003) or as synchrotron emission from a relativistic component, (e.g., Seaquist \& Odegard 1999). Halos can also be observed in soft X-rays, from the diffuse wind fluid (e.g., Strickland and Heckman 2009, M82), and inferred from detection of [CIV] absorption at large impact parameters from starburst galaxies (e.g., Borthakur et al. 2013).

NGC 5128 does not display these canonical signs of a superwind. However, it is not an isolated disk galaxy. It is a large elliptical, with a hot ISM, and it also hosts an AGN which has produced the radio lobes of Cen A. Thus, conditions in NGC5128 may be sufficiently different from the well-known superwind host galaxies to preclude the detection of standard wind identifiers, or the wind signatures may be hidden by AGN phenomena. For instance, except for the singular weather system, NGC 5128 does not show the broad bipolar outflow of warm and cold gas extending several kpc above and below the galaxy. This could be the result of less cold ISM being initially available in the starburst disk, or due to increased stresses on cool clouds as they propagate through the hot ISM of the elliptical galaxy. Furthermore, detection of any radio or $\mathrm{X}$-ray signature from the wind is made difficult by several things: the large angular size of Cen A (which makes both radio and $\mathrm{X}$-ray observing challenging); the bright and complex radio and X-ray structures associated with the $\sim 5 \mathrm{kpc}$ AGN jet; X-ray emission from the hot galactic 
ISM in NGC5128; and interactions between the jet/Inner Lobes and the hot IGM.

Although the typical signatures of a superwind have not been detected in NGC 5128/Cen A, several observational results are suggestive of a wind:

1) Philips et al. (1984) discovered a halo of highly ionized [OIII] emission; they report this emission has velocity dispersion $\sim 350 \mathrm{~km} \mathrm{~s}^{-1}$ in the line of sight, and discuss its likely origin in a biconical outflow - i.e., a wind - from the central disk. The [OIII] is also detected by Bland-Hawthorn and Kedziora-Chudczer (2003). Sharp and Bland-Hawthorn (2010) claim that the [OIII] closely matches extended X-ray emission (reported by Kraft et al. 2008) extending $\gtrsim 1 \mathrm{kpc}$ above and below the gas/dust disk, and $\sim 2 \mathrm{kpc}$ along disk.

2) Emission-line imaging of clouds in the Outer Filament, and extending $\sim 8 \mathrm{kpc}$ beyond the Outer Filament, shows arcs of ionized gas which sit "upstream" (galaxyward) of the clouds. These structures are clearly visible in $\mathrm{H} \alpha$ and NII Maryland Magellan Tunable Filter images of the Outer Filament (Ellis, 2013, private communication ${ }^{6}$ ), and can also be seen, albeit less clearly, in published images (Graham 1998 Figures 2 and 4; Mould et al. 2000 Figure 4; Morganti et al. 1991 Figure 2b). The arcs are very suggestive of bow shocks expected to form upstream of dense clouds sitting in a supersonic flow. These images also show faint streamers of gas trailing $\mathrm{NE}$ of the ionized gas clouds, suggestive of material that has been removed from the clouds and is being carried along downstream by a wind.

3) Some features in X-ray images may reveal the base of the wind. Feigelson et al. (1981) report two elongated $\mathrm{X}$-ray ridges parallel to, but offset from, the dust disk. These are also apparent in recent Chandra images (Kraft et al. 2008 Figure 2, Croston et al. 2009 Figures 2 and 3) ${ }^{7}$ which show a bright, diverging region of X-ray emission, extending above and below the dust disk with sharp edges suggestive of the walls of a superwind. Spectral analysis of one of the walls (Region 3, Croston et al. 2009) finds thermal emission from gas at $\lesssim 1 \mathrm{keV}$, not inconsistent with the relatively cool edges inferred for other winds (e.g., Ranalli et al. 2008).

\subsection{Does the wind reach the transition regions?}

To learn how the wind propagates in and through the Transition Regions, we use the two example winds from Section 5.1. We choose a low-power, pure-starburst wind (at $P_{w} \sim 1 \times 10^{42} \mathrm{erg} \mathrm{s}^{-1}$ and $v_{w} \sim 2000 \mathrm{~km} \mathrm{~s}^{-1}$ ), and a higher-power, AGN-enhanced wind (at $P_{w} \sim 1 \times 10^{43}$ $\operatorname{erg~\mathrm {s}^{-1}}$ and $v_{w} \sim 6300 \mathrm{~km} \mathrm{~s}^{-1}$ ).

Either wind can easily escape the galaxy. Globular cluster kinematics show the gravitating mass of NGC 5128 $\sim 1 \times 10^{12} M_{\odot}$ out to $\sim 40 \mathrm{kpc}$ (Woodley 2010), giving an escape speed $\sim 330 \mathrm{~km} \mathrm{~s}^{-1}$ on that scale. A wind moving

\footnotetext{
6 Images are visible online at www.atnf.csiro.au/research/cena/documents/presentations/ellis.pdf

7 The most detailed image, from Burke et al. (2013), is available online at www.chandra.harvard.edu/photo/2014/cena .
}

at $\gtrsim 2000 \mathrm{~km} \mathrm{~s}^{-1}$ is not seriously restricted by the galaxy's gravity. If the wind flows freely at this speed, in $50 \mathrm{Myr}$ it will reach $\gtrsim 100 \mathrm{kpc}$ from its origin, thus will have no trouble reaching the Transition Regions in the likely life of the central starburst ( $\gtrsim 50 \mathrm{Myr}$; Section 4.2 ).

The wind does not flow into vacuum, however. Its progress will be impeded by thermal pressure of the ambient medium, $p_{o}$, which in this case comes from the ISM of NGC 5128 and tenuous plasma in the large-scale radio lobes. When the ram pressure $\left(\rho_{w} v_{w}^{2}\right)$ of the expanding wind drops to the level of the ambient pressure the wind can no longer expand freely. Instead, it decelerates by means of a termination shock which sits at the distance $R_{\text {shock }}$, where the two pressures balance. Past $R_{\text {shock}}$, the shocked wind plasma forms a hot bubble, slowly expanding into the ambient medium. Immediately past the shock, the wind plasma slows to subsonic flow at $\sim(.3-.4) v_{w}$, and decelerates even further as it approaches the outer edge of the bubble (e.g., Castor et al. 1975).

To estimate the location of the termination shock - and thus whether the wind is likely still to be supersonic when it moves through the Transition Regions - we consider two illustrative geometries. In one case, the wind might expand spherically into an isotropic ISM. Alternatively and likely more relevant for Cen A - the wind may be channeled into the pre-evacuated Outer Radio Lobes.

Spherical expansion into ISM - If there were no preexisting radio lobe, the wind would expand spherically into the undisturbed galactic ISM at density $10^{-3} \mathrm{~cm}^{-3}$ and pressure $\sim 8.5 \times 10^{-13} \mathrm{dyn}_{\mathrm{cm}^{-2}}$ (revealed by X-ray data; Kraft et al. 2009). In this situation, the termination shock sits at a position given by $R_{\text {shock }}^{2} \simeq P_{w} / 4 \pi p_{o}$. In a pure-starburst wind the shock sits at only $R_{\text {shock }} \sim 7$ kpc from the core; in an AGN-enhanced wind it sits at $R_{\text {shock }} \sim 13 \mathrm{kpc}$ from the core. Thus, by the time the wind plasma reaches the Transition Regions, it carries the same amount of power, but becomes a subsonic breeze rather than a supersonic wind.

Wind channeled into radio lobes - The situation in Cen A is likely to be more complicated. The low-density radio lobes (previously evacuated by the AGN) may constrain the wind to flow within a relatively small solid angle, $\Omega \ll$ $4 \pi$. If this is the case - and if the wind flow remains laminar within the channel - the termination shock sits at a position given by $R_{\text {shock }}^{2} \simeq P_{w} / 8 \pi f p_{o}$, where $f=\Omega / 4 \pi$, and we assume half of the total power goes to each side of the galaxy. Based on radio images, we estimate the wind has expanded laterally to a width of $\sim 24 \mathrm{kpc}$ when it is $30 \mathrm{kpc}$ from the galaxy in the North Transition Region. Thus, the "open channel" occupies only a fraction $f \sim 5 \%$ of the full solid angle that would be available to a wind expanding spherically into an isotropic medium.

To estimate pre-wind conditions in the radio lobe, we follow Eilek (2014), who argued that the Outer Lobes on large scales are in pressure balance with the local IGM (thus the pressure drops from $\sim 8.5 \times 10^{-13} \mathrm{dyn}^{-2}$ close 
to the galaxy, to $\sim 3.2 \times 10^{-13} \mathrm{dyn}_{\mathrm{cm}}^{-2}$ past $\sim 100 \mathrm{kpc}$ from the galaxy). In this scenario, the termination shock of a pure-starburst wind sits at $R_{\text {shock }} \sim 30 \mathrm{kpc}$ from the core, nearly reaching the outer end of the weather ribbon. In an AGN-boosted wind, the shock sits at $R_{\text {shock }} \sim 65$ kpc. Thus if the galactic wind is channeled into the lowdensity radio lobes, it may still be supersonic when it reaches the North and South Transition Regions.

Could we see the termination shock? - Finally, one might ask if these termination shocks could be detected observationally. Detection in X-rays seems unlikely; the density jump in such tenuous plasma would be very faint, and X-ray imaging would be severely challenged by the large angular size, and variations in Galactic foreground emission. One might also expect the shock to be radio-bright, due to particle acceleration and magnetic field amplification local to the shock. Eilek (2014) has in fact suggested that the bright filaments within the outer lobes (seen in ATCA image from Feain et al.2011) could be internal shocks, driven by transonic outflow within the lobes. The complex nature of the radio-bright filaments makes it difficult to identify any one structure as "the" terminal wind shock; but if the wind flow becomes turbulent as it propagates into the Outer Lobes, it may be the origin of the observed radio-bright filaments.

\section{DISCUSSION: THE WIND CAN DRIVE THE WEATHER}

A wide range of phenomena in the North Transition Region require ongoing energization: emission-line filaments, young stars, radio and X-ray knots, and the radioloud North Middle Lobe itself. In addition, energy flow through the North and South Transition Regions is needed to keep the Outer Lobes shining in radio and $\gamma$-rays. Although there seem to be no collimated jets in either Transition Region, we have argued that a wind from the starburst, perhaps augmented by the AGN, is flowing through both regions right now. In this section, we explore how the wind in NGC 5128 can create or sustain these weatherrelated phenomena.

\subsection{Connecting the wind to emission-line clouds}

Several authors have suggested that a jet flowing past dense, cold gas clouds can energize the clouds, producing the weather phenomena in Cen A. We argue here that a supersonic wind can have the same effect. We follow previous authors who have considered the behavior of cold dense clouds in a starburst wind, and apply their work to the specific context of Cen A.

Induced star formation - Although the detailed physics remains unclear, it has often been suggested that plasma flow past cold clouds will induce star formation in the clouds (e.g., Fragile et al. 2004, Gaibler et al. 2012). The flow could, or course, be either a jet or a wind. The "typical emission-line cloud" we use in Section 3.4 - to represent the emission line clouds studied by Morganti et al. (1991) - is not gravitionally unstable. However, we can speculate that some of the clouds, when perturbed and/or fragmented by the passing flow, will cool rapidly enough to become unstable and collapse to form stars (e.g., Mellema et al.2002, Cooper et al.2009).

Bow shocks around the clouds - When a supersonic wind encounters slower clouds, bow shocks will form upstream of the clouds. Some clear examples are apparent in simulations from Cooper et al. (2009). H $\alpha$ images of the Outer Filament in Cen A strongly suggest that such bow shocks exist upstream of the emission-line clouds there (Section 5.2 . This has several interesting consequences for the local weather. (1) The upstream ram pressure, moderated by the bow shock, will help confine the high-pressure emission-line clouds and radio/X-ray knots (if the latter are diffuse gas). (2) The hot shocked wind plasma around the clouds will be an X-ray source (e.g., Marcolini et al. 2005, Cooper et al. 2009), consistent with the observed spatial correlation of X-rays with some emission line clouds. (3) The shocks are likely to accelerate electrons to relativistic energies, and also enhance the local magnetic field (as we discuss in Section 6.3), consistent with the observed spatial correlation of nonthermal radio emission with the $\mathrm{FUV} / \mathrm{H} \alpha$ ribbon.

Sources for the gas in the weather ribbon - In Section 3.3 . we suggested that cold HI close to NGC 5128 - in particular the "17-kpc cloud" and smaller structures closer to the galaxy (Figure 6) - might be the source of the material in the extended weather ribbon. If this is the case, gas clouds torn from the parental HI clouds must maintain their identity for at least $50 \mathrm{Myr}$, the travel time from the $17-\mathrm{kpc}$ cloud to the end of the weather ribbon. This may be difficult, because the clouds are subject to destructive forces as they are carried outwards by a faster, hot galactic wind. They can be be evaporated by thermal conduction as they sit in the hot wind. They can also be shredded by the Kelvin-Helmholtz instability as the hot wind flows past them.

To check this, we estimate lifetimes for our typical emission-line clouds (Section 3.4 against both effects (details and references are given in the Appendix). We find the answer is uncertain. Simple estimates suggest the clouds are destroyed very rapidly, in only a few Myr. This seems to imply that HI closer to NGC 5128 cannot be the only source of material in the weather ribbon. However, recent simulations identify mitigating effects which may slow down the destruction, and retain the cloud's identity, for longer than the lifetimes predicted by the simple arguments.

Thus, while it is possible that known HI clouds can be the source for the entire weather ribbon, the question is not settled. Other more local mechanisms may be necessary. Some of the gas in the extended weather system may have come from other cold clouds - not yet detected, or perhaps now destroyed - which happen to lie farther than $17 \mathrm{kpc}$ from the galaxy and in the path of the wind. Another possibility is that star formation in 
the weather ribbon close to the galaxy created young star clusters which continue to move outwards at the speed of their parental gas clouds. Winds from these young clusters, driven by normal stellar evolution, may supply new gas to the weather system which we now detect as part of the $\mathrm{FUV} / \mathrm{H} \alpha$ ribbon.

\subsection{Energy budget for the clouds}

There are many ways to energize cool clouds within a hot, supersonic wind. The wind's ram pressure can drive shocks into a cloud, heating the cloud as the shocks dissipate. If star formation is induced within the cloud, young massive stars will photoionize their environs. Thermal conduction will heat the cloud. Ambient shear flow, coupled to the cloud by the Kelvin-Helmholtz instability, will drive random motions and turbulence that heat the cloud when they dissipate.

Detailed modelling of these complex processes is beyond the scope of this paper, but we can constrain the energy budget. Following Sutherland et al. (1993), we consider emission-line clouds in the Inner and Outer Filaments. The very short cooling time of these clouds (Table 2) means they need ongoing energization, which must ultimately come from the wind flow. Taking the $\mathrm{H} \beta$ power for a large cloud complex (collection of small, 10-pc cloudlets; Section 3.4 in the Inner and Outer Filaments as $L_{H \beta} \sim$ $10^{37} \mathrm{erg} \mathrm{s}^{-1}$ (Morganti et al. 1991), and estimating the total line power $\sim(50-100) L_{H \beta}$ (Sutherland et al. 1993), we see the wind must supply $\sim(5-10) \times 10^{38} \mathrm{erg} \mathrm{s}^{-1}$ to each such cloud complex. We estimate the wind flow has $\sim 6 \mathrm{kpc}$ radius when it encounters the Inner and Outer Filaments, and take $\sim(200 \mathrm{pc})^{2}$ as the effective area of the cloud complex seen by the incoming wind.

With these numbers, a pure-starburst wind running at $5 \times 10^{41} \mathrm{erg} \mathrm{s}^{-1}$ (to each side of the galaxy) would convey $\sim 2 \times 10^{38} \mathrm{erg} \mathrm{s}^{-1}$ to the cloud complex. This is not enough to maintain the line luminosity, even if all of the incoming wind energy is efficiently transferred to the line emission. More energy is needed - and it is available if the starburst wind is enhanced by energy from the AGN. For instance, if the AGN boosts the wind power by a factor of ten, the cloud complex would see $\sim 2 \times 10^{39} \mathrm{erg} \mathrm{s}^{-1}$ - enough to keep the clouds stirred up and maintain their emissionline power.

\subsection{Connecting the wind to the radio emission}

The North and South Transition Regions are asymmetric. The North Transition Region contains the dramatic weather ribbon, seen in optical, UV and X-rays, but the South Transition Region contains no similar feature. We have argued that a bipolar wind from the core of the galaxy creates the weather ribbon in the North Transition Region when it energizes cold gas that happens to lie in its path. By contrast, the lack of a similar weather system in the South Transition Region must be due to to a lack of cold gas clouds in the wind's southern flight path.
Why is the North Middle Lobe radio bright? - The North Transition Region contains the diffuse, radio-bright North Middle Lobe as well as the radio-loud knotty ridge that coincides with the optical/FUV/X-ray weather system. Synchrotron radio emission from the region will be enhanced when the interaction of the supersonic wind with the cold gas generates shocks (as the wind is forced to change direction and/or decelerate) and MHD turbulence (easily generated by any perturbation to an MHD flow). Shocks can quickly energize relativistic particles and amplify magnetic fields (e.g., Drury 1983, Schure et al. 2012). Alfven waves within MHD turbulence can also accelerate relativistic particles (e.g., Lacombe 1977, Eilek 1979).

If this is the case, the radio-bright ridge and knots coincident with the weather system are due to localized particle acceleration and field amplification where the wind encounters cool gas in the weather system. The enhanced radio surface brightness which continues to the northwest - and defines the larger North Middle Lobe - could be due to advection of shock-accelerated plasma within the wind and/or local particle acceleration due to MHD turbulence in the wind.

Why no equivalent South Middle Lobe? - No comparable radio structure has been detected in the South Transition Region, which contains only weak, diffuse radio emission (perhaps a factor $\sim 10$ fainter than in the NML; Paper 1). If the radio-brightness of the North Middle Lobe is due to to the interaction of the galactic wind with cold gas clouds which happen to be in the region, then the asymmetry of the North and South Transition regions must be due to the lack of corresponding cold HI to the south of the galaxy. We therefore suggest that the radio-faint South Transiton Region region shows the basic, unperturbed wind flow. That is, some relativistic, magnetized plasma (from the starburst, possibly enhanced by the AGN) is advected out with the wind. It radiates faintly as it goes, but its radio emission is not amplified by any interaction with cold gas clouds in its path.

In contrast to the North Middle Lobe, which we have argued is at a higher pressure than the ambient galactic ISM (Paper 1, also Section 3.4), there is no evidence that the radio-loud plasma in the South Transition Region (STR) is over-pressured. In Paper 1 we found that the minimum pressure in the radio-loud North Middle Lobe is a factor $\sim 4$ larger than that of the ambient ISM. Because in Paper 1 we could only put upper limits on the radio power from the STR, we could not estimate its volume emissivity (which is required to calculate the minimum pressure; e.g., Paper 1 Appendix). We can, however, use the lowerresolution image of Junkes etal (1993; kindly provided by N. Junkes) to estimate the mean surface brightness in the STR to be $\sim 10 \%$ of that in a comparable area of the NTR. Because the minimum pressure is approximately proportional to the square root of the surface brightness, it is a factor $\sim 3$ lower, on average, in the STR than in the NTR. We therefore argue that current observations are consistent with the South Transition Region being in 
pressure balance with the ambient ISM - and thus with the South Transition Region not being energized by interaction with dense gas clouds in its path.

This argument requires a particular geometry for the cold HI clouds shown in Figure 6. If the gas in the northern weather ribbon is indeed being dragged out of the 17kpc HI cloud by a wind (which we envision as filling a flaring cylindrical region above and below the starburst disk), most of that cloud must be located outside the wind; otherwise it would seed warm emission-line gas everywhere along its length. Thus, the observed arc of HI clouds must be either in front of, or behind, the suspected wind flow. Since only one edge of the $17-\mathrm{kpc}$ cloud appears to be impacted by the wind, that edge of the cloud must be rotating into the wind flow. Figure 6 shows there is also an HI cloud SW of the galaxy. If this southwestern cloud were located in the wind flow, we would expect to detect a Southern Middle Lobe and an equivalent southern weather system. Taken together, these arguments suggest the southwestern HI cloud, and most of the ring of HI clouds (e.g.to North and Northwest of center), are well away from the wind flow.

\subsection{Energy budget for the radio emission}

Detailed modelling of the complex shock/turbulence physics leading to enhanced synchrotron emission is beyond the scope of this paper, but we can check the energetics. The radio-bright North Middle Lobe has excess pressure $\gtrsim 4 \times 10^{-12} \mathrm{dyn}^{-2}$ (Paper 1 , assuming the baryon/lepton ratio there is similar to that in our Galaxy). Taking the measured area of the North Middle Lobe as a cylinder with radius $12 \mathrm{kpc}$ and length $30 \mathrm{kpc}$, the excess energy is $3 p V \gtrsim 5 \times 10^{57} \mathrm{erg}$. We have argued that this excess energy comes from the galactic wind encountering cold gas in the North Transition Region which happens to lie in the wind's path.

If the galactic wind is driven only by the central starburst, it must have continued at its current power for 300 Myr in order to provide the extra energy, or even longer if some of the wind energy continues past the Transition Regions to power the Outer Lobes. While it is not impossible for the central starburst to have continued so long, the much shorter timescales of North Middle Lobe phenomena ( 15-30 Myr; Table 2 $)$ suggest that higher energy input rates may be required. In addition, we find it unlikely that the AGN would have remained "off" for such a long period (a substantial fraction of the dynamic age of the Outer Lobes; Section 1.1, also discussion in Paper $1)$.

Alternatively, if the AGN has boosted the wind power by a factor of ten (our example in Section 4.3), that wind provides enough energy to create the North Middle Lobe in only $30 \mathrm{Myr}$ (or longer if some wind energy continues into the Outer Lobes). Because the starburst has lasted for at least $50 \mathrm{Myr}$, and probably longer, we see that an AGN-augmented wind easily is able to energize the North Middle Lobe, even if some of its energy continues on into the Outer Lobes. This scenario fits well with the frequent re-energization of shocks and/or turbulence in the Outer Lobes (Section 1.1) needed to keep them shining in radio and $\gamma$-rays. That energy must have come from the inner galaxy, by way of the North and South Transition Regions. Although there seem to be no jets at present within the Transition Regions, a diffuse wind can play the same role, carrying energy and mass from the galactic core into the large-scale radio lobes.

\section{SUMMARY}

In this paper we have presented new GALEX observations which change and enhance our picture of the circumgalactic environment around NGC 5128 / Cen A.

- The North Transition Region is an interesting place. In addition to the radio-loud North Middle Lobe, deep FUV and $\mathrm{H} \alpha$ images show that a complex weather ribbon - containing a mix of young stars, emission-line clouds, bright X-ray knots and a knotty radio-loud ridge - extends $\gtrsim 35 \mathrm{kpc}$ into the North Transition Region. Most features in this weather system are evanescent, with typical lifetimes only a few Myr. Because the NTR is almost certainly older than this, some in situ driver must be reenergizing the weather phenomena.

- The inner galaxy contains two energy sources: a starburst and an AGN. Our GALEX data reveal a starburst in the central dust/gas disk, with star formation rate $\sim 2 M_{\odot} \mathrm{yr}^{-1}$. It has been going for at least $\sim 50 \mathrm{Myr}$, and probably - based on other well-studied starbursts for more than 100 Myr. It seems likely that the AGN has co-existed with the starburst for at least part of the latter's life, probably adding its power to the overall energy budget of the system.

- A galactic wind is blowing through both Transition Regions right now. Although there seem to be no collimated jets in either Transition Region (i.e.beyond $\sim 7 \mathrm{kpc}$ from the core), the central starburst should be driving out a wind. Unless the AGN has been inactive for the duration of the starburst - which we deem unlikely - energy and mass driven out from the AGN has probably contributed to the wind. The resulting enhanced galactic wind will carry mass and energy into and through the Transition Regions.

- The wind can drive the weather in the North Transition Regions. This galactic wind can energize and stimulate star formation in dense gas clouds it encounters in the North Transition Region, thus both causing and maintaining the extended weather system. The interaction of the wind with dense gas clouds can also accelerate relativistic electrons, causing the radio-loudness of the North Middle Lobe. The lack of any comparable weather system or enhanced radio emission to the south does not mean there is no flow in the South Transition Region, but rather that no dense gas clouds happen to sit in the southern wind's flow path.

At some level, the difference between an AGNaugmented wind and a wind-enhanced jet is largely a semantic one, probably depending on the details of the 
interaction between the two. Further spectroscopic imaging observations, and perhaps polarization imaging, particularly of the striking FUV and $\mathrm{H} \alpha$ ribbon, would be invaluable in confirming the existence of the starburst wind, and would allow us to gain significant insight into energy transport in this nearby active galaxy. It may well be that such a combined outflow is a common property of lowluminosity AGN, but we are not able to detect the combination in more distant systems. Future understanding of Cen A, the nearest active galaxy, will require consideration of the influences of an active starburst and wind on the system.

We are very grateful to I. Evans, R. Kraft, D. Schiminovich, and N. Junkes for sharing their data with us. We appreciate discussions about ongoing work with R. Sharp and S. Ellis. Thanks to E. Greisen for friendly software support throughout this project, and to D. Thilker, S.
Wykes, J. Marvil and J. Ott for stimulating conversations. SGN thanks the NRAO in Socorro, NM, for hospitality during major parts of this work.

SGN is very grateful to Chris Martin and the GALEX team. This work is based on observations made with the NASA Galaxy Evolution Explorer (GALEX), which was operated for NASA by the California Institute of Technology under NASA contract NAS5-98034. In this work we have made extensive use of both NASA's Astrophysics Data System (ADS; hosted by the High Energy Astrophysics Division at the Harvard Smithsonian Center for Astrophysics), and the NASA/IPAC Extragalactic Database (NED; operated by the Jet Propulsion Laboratory, California Institute of Technology, under contract with NASA)

We appreciate thoughtful comments from the referee, which have helped us improve the paper.

\section{APPENDIX}

\section{HOW LONG CAN THE EMISSION-LINE CLOUDS LAST?}

Dense, cool clouds moving relative to a hot ambient medium are subject to disruption by the Kelvin-Helmoltz (KH) instability as well as ablation by thermal conduction. If the structures in the weather system have come from HI clouds close to NGC 5128, they must be able to survive the trip in the face of this adversity. In this Appendix we estimate these timescales and discuss possible mitigating effects which might enhance the cloud's longevity. For numerical purposes we use our "typical" emission-line cloud (density $n_{c} \sim 10 \mathrm{~cm}^{-3}$, temperature $T_{c} \sim 10^{4} \mathrm{~K}$, size $a_{c} \sim 10 \mathrm{pc}$; Section 3.4.

\section{Simple calcuations suggest rapid destruction}

Our typical line-emitting cloud will evaporate when thermal conduction transfers heat from the ISM into the cloud core. Because the Coulomb mean free path is short for our cloud, classical heat flux applies. Following Cowie \& McKee (1977), also Marcolini et al. (2005), the evaporation time for a cloud of radius $a_{c}$ and density $n_{c}$ can be written $t_{\text {evap }} \sim 2 n_{c} a_{c}^{2} k_{B} / \kappa$, where $k_{B}$ is the Boltzmann constant, and the thermal conductivity $\kappa$ contains the important physics. If $\kappa$ is given by the Spitzer value (e.g., Cowie \& McKee 1977), and the ambient ISM at $T \sim 10^{7} \mathrm{~K}$, we expect the cloud to evaporate in only $t_{\text {evap }} \sim 2$ Myr.

If our cloud is in motion relative to the ambient medium, it is also subject to the Kelvin-Helmholtz instability, which will heat and fragment the cloud, and thus cause its dispersal (e.g., Klein et al. 1994). The timescale for this instability to disrupt a cloud of radius $a_{c}$, moving relative to the ambient medium at some $\Delta v$, is $t_{K H} \sim a_{c}\left(n_{c} / n_{w}\right)^{1 / 2} / \Delta v$, where $n_{w}$ is the density of the local wind plasma (e.g., Fragile et al. 2004). If our emission-line-cloud sits in a pure-starburst wind (as in Section 5.1), we estimate $\Delta v \sim 2000 \mathrm{~km} \mathrm{~s}^{-1}$ and $n_{w} \sim 5 \times 10^{-5} \mathrm{~cm}^{-3}$ (estimating a 6-kpc flow radius for the wind close to the $17-\mathrm{kpc}$ HI cloud). Under these conditions, $t_{K H} \sim 2.2 \mathrm{Myr}$. If the AGN boosts the wind by a factor of ten (as in Section 5.1, with $\Delta v \sim 6300 \mathrm{~km} \mathrm{~s}^{-1}$, a similar calculation gives $n_{w} \sim 2 \times 10^{-5} \mathrm{~cm}^{-3}$, and $t_{K H} \sim 1$ Myr.

\section{Possible extensions of cloud life}

Both the Kelvin-Helmholtz and thermal ablation timescales are significantly smaller than the $\sim 50$ Myr cloud transit time from the 17-kpc HI cloud to the end of the weather ribbon (Section 3.3). This appears to challenge the possibility that the weather system material originated in the $17-\mathrm{kpc} \mathrm{HI}$ cloud, or from other HI closer to the galaxy. However, uncertainties in the models may extend the effective lifetime of the cloud.

One important uncertainty is the actual value of thermal conductivity in the North Transition Region. Observations of galaxy clusters suggest $\kappa$ may be significantly lower than the Spitzer value (e.g., Churazov etal 2001, Narayan \& Medvedev 2001). This is consistent with many other lines of evidence supporting collisionless transport effects, as well as thermal isolation of the clouds by magnetic fields in the cloud-ISM interface. Because the relevant physics is far from understood, we do not attempt to estimate an alternative $t_{\text {evap }}$, but just note it may be significantly longer than the classical estimate.

Another uncertainty is the evolution of a Kelvin-Helmholtz-unstable cloud. Recent work identifies effects which may lengthen the cloud lifetime. Marcolini et al. (2005) find the temperature gradient set up by thermal conduction will 
slow KH growth. In addition, if a cloud can radiate fast enough to offset shear-induced heating, Cooper et al. (2009) find that KH-stripped material will cool to $\sim 10^{4} \mathrm{~K}$ and accumulate in smaller cloudlets which are drawn into the downstream flow to form filaments of cool (emission-line) gas. In the simulations these cloudlets can last several times longer than the linear KH instability time.

In Cen A, the combination of these two effects may lead to the cool cloudlets keeping their identity as emission-line clouds for long enough to reach the end of the weather system. However, because current simulations neither extend to such long timescales, nor track the eventual fate (such as fragmentation, evaporation, star formation) of KH-stripped cloudlets, we cannot say with certainty that the 17 -kpc HI cloud is the source of the entire extended weather system.

\section{REFERENCES}

Abdo, A., et al. Fermi-LAT Collaboration, 2010, Science, 328, 725

Armus, L., Heckman, T. M., Weaver, K. A. \& Lehnert, M. D., 1995, ApJ 445, 666.

Auld, R., Smith, M.W.L., Bendo, G., et al., 2012, MNRAS 420, 1882

5Bicknell, G. V. 1984, ApJ 286,68

Bland, J., Taylor, K., \& Atherton, P. D., 1987, MNRAS 228, 595

Bland-Hawthorn, J \& Kedziora-Chudczer, L., 2003, PASA 20, 242

Bolton, J. G., Stanley, G .J., \& Slee, O. B., 1949, Nature 164, 101

Borthakur, S., Heckman, T., Strickland, D., et al., 2013, ApJ 768, 18

Burke, M. J., Raychaudhury, S, Kraft, R. P. et al., 2012, ApJ 766, 88.

Burns, J. O., Feigelson, E. D., \& Schreier, E. J. 1983, ApJ 273, 128

Canning, R. E. A., Ryon, J. E., Gallagher, J. S. et al., 2014, MNRAS $444,336$.

Castor, J., McCray, R. \& Weaver, R., 1975, ApJ 200, L107

Charmandaris, V., Combers, F. \& van der Hulst, J. M., 2000, A\&A, 356,1

Clarke, D. A., Burns, J. O., \& Norman, M. L. 1992, ApJ 395, 444.

Chevalier, R. A. \& Clegg, A. W., 1985, Nature, 317, 44.

Churazov, E., Brüggen, M. Kaiser, C. R. et al. , 2001, ApJ 554, 261

Cooper, J.L., Bicknell, G. V., Sutherland, R. S., \& Bland-Hawthorn, J., 2008, ApJ 674, 157.

Cooper, J. L., Bicknell G. B., Sutherland, R. S.\& Bland-Hawthorn, J., 2009, ApJ 703, 330.

Cowie, L. L. \& McKee, C. F., 1977, ApJ 211, 135

Crnojevic D., Grebel E. K. \& Cole, A. A., 2011, A\&A 530, A59

Crockett, R. M., Shabala, S. S., Krviraj, S., et al., 2012, MNRAS 421,1603

Croft, S., Van Breugel, W., de Vries, et al., 2006 ApJ 647, 1040.

Croston, J.H., Kraft, R.P., Hardcastle, M.J., et al., 2009, MNRAS $395,1999$.

Drury, L. O'C., 1983, Reports on Progress in Physics, 46, 973

Dufour, R. J. \& van den Bergh, S., 1978, ApJLett 226, L73

Dufour, R. J., van den Bergh, S., Harvel, et al., 1979, AJ 84, 284

Dunlop, J., 1828, Philosophical Transactions of the Royal Society, 118,113

Eilek, J A., 1979, ApJ 230, $373 \mathrm{~S}$

Eilek, J. A., 2014, New Journal of Physics 16, 045001

Everett, J. E., Zweibel, E.G., Benjamin, R. A. et al. , 2008, ApJ 674 258

Evans, I. \& Koratkar, A. 2004, ApJ 617, 209

Fabian, A. C. 2012, ARAA 50, 455

Feain, I.. J., Cornwell, T.J., Ekers, R. D., et al. 2011, ApJ 740, 17

Feigelson, E. D., Schreier, E. J., Delvaille, et al., 1981, ApJ 251, 31. 363, 91

Fomalont, E. G., Ebneter, K. A., van Breugel, W. J. M. \& Ekers, R. D., 1989, ApJ 346, L17

Förester Schreiber, N. M., Genzel, R., Lutz, D. \& Kunze, D., 2001, ApJ 552, 544

Fragile, P. C., Murray, S. D., Anninos, P., \& van Breugel, W., 2004, ApJ 604, 74

Gaibler, V, Khochfar, S, Krause, M \& Silk, J, 2012, MNRAS 425, 438

Gil de Paz, A., Boissier, S, Madore, B. F. et al. 2007, ApJS 173, 185

Goodger, J. L., Hardcastle, M. J., Croston, J. H., et al. 2010, ApJ 708,657

Graham, J. A., 1983, ApJ 269, 440

Graham, J. A., 1998, ApJ 502, 245

Graham, J. A., and Fassett, C. I., 2002, ApJ 575, 712

Graham, J. A. and Price, R. M., 1981, ApJ 247, 813

Hardcastle, M. J., Worrall, D. M., Birkinshaw, M., et al. 2002, MNRAS, 334, 182

Hardcastle, M. J., Worrall, D. M., Kraft, R. P., et al., 2003, ApJ 593,169
Hardcastle, M. J., Cheung, C. C., Feain, I. J. \& Stawarz, L., 2009 MNRAS 393, 1041

Harris, G. L. H., Rejkuba, M., \& Harris, W. E., 2009, PASA 27, 457.

Heckman, T. M., Dahlem, M., Lehnert, M. D., et al., 1995 ApJ 448, 90

Helou, G., Soifer, B. T. \& Rowan-Robinson, M., 1985, ApJ 298, L7

Herschel, J. F. W., 1847, Results of Astronomical Observations at the Cape of Good Hope (London: Smith, Elder)

Hoopes, C. G., Heckman, T. M., Strickland, D. K., et al. , 2005, ApJ 619, L99

Junkes, N., Haynes, R. F., Harnett, J. I., \& Jauncey, D.L. 1993, A\&A269, 29.

Kennicutt, R. C. Jr, 1998, ARAA 36, 189

Klein, R. I., McKee, C. F. \& Colella, P., 1994, ApJ 420, 213

Kraft, R. P., Hardcastle, M. J. Sivakoff, G. R., et al., 2008, ApJ 677, L97

Kraft, R. P., Forman, W. R., Hardcastle, M. J., et al., 2009, ApJ $698,2036$.

Lacombe, C., 1977, A\&A 54, 1

Lehnert, M. D. \& Heckman, T. M., 1996, ApJ 462, 651

Marcolini, A., Strickland, D. K., D'Ercole, A., et al., 2005, MNRAS 362,626

Marconi,A., Schreier, E. J., Koekemoer, A. et al., 2000, ApJ 528, 276.

Martin, D. C., Fanson, J., Schiminovich, D., et al., 2005, ApJ 619, L1

Marvil, J. S., Owen, F. N \& Eilek, J A., 2014, arXiV 1408.6296, to appear in AJ.

McQuinn, K. B. W., Skillman, E. D., Cannon, J. M. et al., 2010, ApJ 724,49

McQuinn, K. B. W., Skillman, E. D., Cannon, J M et al., ApJ 2009, 695,561

Mellema, G., Kurk, J. D. \& Rottgering, H. J. A., 2002, A\&A 395, L13.

Minniti, D., Rejkuba, M., Funes, J. G. \& Kennicutt, R. C., 2004, ApJ 612, 215

Morganti, R., Robinson, A., Fosbury, R. A. E., et al. 1991, MNRAS $249,91$.

Morganti, R., Fosbury, R.A.E., Hook, R. N., et al., 1992, MNRAS $256,1 \mathrm{P}$.

Morganti, R., Kileen, N. E. B., Ekers, R. D., \& Osterloo, T. A. 1999, MNRAS 307, 750 .

Morrissey, P. M., Conrow, T., Barlow, T. A., et al. , 2005, ApJSupp 173,682

Mould, J. R., Ridgewell, A., Gallagher, J. S., et al. , 2000, ApJ 536, 266.

Murphy, E. J., Condon, J. J., Schinnerer, E., et al., 2011 ApJ 737, 67

Narayan, R. \& Medvedev, M. V., 2001, ApJ 562, L129

Nicholson, R. A., Bland-Hawthorn, J. \& Taylor, K. 1992, ApJ 387, 503

Neff, S. G., Eilek, J. A. \& Owen, F. N., 2015, to appear in ApJ (Paper 1)

Osterloo, T. A., \& Morganti, R. 2005, A\&A 429, 469

Parkin, T. J., Wilson, C D, Foyle K etal, 2012, MNRAS 422, 2291

Peng, E. W., Ford, H. C. \& Freeman, K. C., 2004, ApJ, 602, 685.

Phillips, M.M., Taylor, K., Axon, D. J. \& Atherton, P. D. 1984, Nature 310, 554

Quillen, A. C., Graham, J. R., and Frogel, J. A., 1993, ApJ 412, 550

Ranalli, P., Comastri A., Origlia, L \& Maiolino, R., 2008, MNRAS 386,1464

Rejkuba, M., Minniti, D., Courbin, F. \& Silva, D. R. 2002, ApJ 564, 688

Rejkuba, M., Harris, W. E., Greggio, L. \& Harris, G. L. H., 2011, A\&A 526, 123

Rice, W, Lonsdale, C J, Soifer B T et al. , ApJ Supp 68, 191 
Rich, J. A. Dopita, M. A., Kewley L. J. \& Rupke, S.D.N, 2010, ApJ 721,505

Saxton, C. R., Sutherland, R. S. \& Bicknell, G. V.. 2001, ApJ, 563, 103.

Seaquist, E. R. \& Odegard, N., 1991, ApJ 369, 320

Schiminovich, D., van Gorkom, J. H., van der Hulst, J. M., \& Kasow, S. 1994, ApJ, 423, L101. H

Schure, K. M., Bell, A. R., Drury, L. O'C. \& Bykov, A. M., 2012, Space Sci REv 173, 491

Sharp, R. G. \& Bland-Hawthorn, J., 2010, ApJ 711, 818

Soria, R., Mould, J. R., Watson, A. M., et al. 1996, ApJ, 465, 79

Sparke, L. S. 1996, ApJ 473, 810

Sparks, W. B., Donahue, M., Jordan, A. et al., 2004, ApJ 607, 294

Steigman, S. G., Strittmatter, P. A., \& Williams, R. E., 1975, ApJ 198,575

Strickland, D. K., Hornschemeier, A., Ptak, A. et al., 2009, Astro2010: The Astronomy and Astrophysics Decadal Survey, Science White Papers, no. 289

Strickland, D. K. \& Heckman,T. M. 2009, ApJ 697, 2030.

Struve, C., Oosterloo, T. A., Morganti, R., Saripalli, L., 2010, A\&A 515, A67.
Sugai, H., Davies, R. I. \& Ward, M. H. J., 2003, ApJ 584, L9

Sutherland, R. S., Bicknell, G. V. \& Dopita, M. A. 1993, ApJ 414, 510.

Tingay, S. J., Preston, R. A., and Jauncey, D. L., 2001, AJ 122, 1697 Unger, S. J., Clegg, P. E., Stacey, G. J. et al. 2000, A\&A 355, 885 van Breugel, W. \& Fomalont, E. G. 1984, ApJ 282, 55

van den Bergh, S., 1976, ApJ 208, 673

Veilleux, S., Cecil, G. \& Bland-Hawthorn, J., 2005, ARAA 43, 769

Veilleux, S. \& Rupke, D. S. 2002, ApJ 565, L63

Veilleux, S., Bland-Hawthorn, J., Cecil, G., Tully, R. B., Miller, S. T 1999, ApJ 520, 111

Viegas, S. M. \& Prieto, M. A. 1992, MNRAS 258, 483

Werner, N., Oonk, J. B. R., Canning, R. E. A. et al., 2013, ApJ 767,153

Westmoquette, M S., Smith L J \& Gallagher, J S III, 2011, MNRAS 414,3719

Woodley, K. A., Harris, W. E., Puzia, T. H., et al., 2010, ApJ 708, 1335.

Yun, M. S., Reddy, N. A. \& Condon, J. J., 2001, ApJ 554, 803 